\title{
DISLOCATION DYNAMICS IN CRYSTALS: A MACROSCOPIC THEORY IN A FRACTIONAL LAPLACE SETTING
}

\author{
SERENA DIPIERRO, GIAMPIERO PALATUCCI, AND ENRICO VALDINOCI
}

\begin{abstract}
We consider an evolution equation arising in the Peierls-Nabarro model for crystal dislocation. We study the evolution of such dislocation function and show that, at a macroscopic scale, the dislocations have the tendency to concentrate at single points of the crystal, where the size of the slip coincides with the natural periodicity of the medium. These dislocation points evolve according to the external stress and an interior repulsive potential.
\end{abstract}

\section{Contents}

1. Introduction

2. Physical motivations

3. Heuristics on the dynamics of the transition layers

4. A comparison principle

5. Layer solution and corrector

6. Auxiliary layer solutions

7. Decay improvement of the solution layer

8. Proof of Theorem 1.1

8.1. Construction of sub and supersolutions

8.2. Existence and uniqueness of the solution

8.3. Convergence

References

\section{INTRODUCTION}

We consider the problem

$$
v_{t}=L_{s} v-W^{\prime}(v)+\sigma_{\epsilon}(t, x) \quad \text { in }(0,+\infty) \times \mathbb{R},
$$

2010 Mathematics Subject Classification. 35Q99, 35B40, 35J25, 35D30, 35G25, 70F99.

Key words and phrases. Nonlinear problems, nonlocal Allen-Cahn equation, reaction-diffusion, Peierls-Nabarro model, dislocation dynamics, particle systems, fractional Laplacian, fractional Sobolev spaces.

Acknowledgements. The second author has been supported by PRIN 2010-11 "Calcolo delle Variazioni". The third author has been supported by ERC grant 277749 "EPSILON Elliptic Pde's and Symmetry of Interfaces and Layers for Odd Nonlinearities". All the authors would like to thank Régis Monneau for his visit to the University of Rome Tor Vergata and the stimulating discussions about dislocation dynamics. 
where $s \in(1 / 2,1), L_{s}$ is the so-called fractional Laplacian and $W$ is a 1-periodic potential. More explicitly, given $\varphi \in C^{2}(\mathbb{R}) \cap L^{\infty}(\mathbb{R})$ and $x \in \mathbb{R}$, we define

$$
L_{s} \varphi(x)=\frac{1}{2} \int_{\mathbb{R}} \frac{\varphi(x+y)+\varphi(x-y)-2 \varphi(x)}{|y|^{1+2 s}} \mathrm{~d} y .
$$

We refer to $[21,8]$ for a basic introduction to the fractional Laplace operator. As for the potential, we assume that $W \in C^{2, \alpha}(\mathbb{R})$, for some $0<\alpha<1$, and:

$$
\left\{\begin{array}{l}
W(u+1)=W(u) \quad \text { for any } u \in \mathbb{R}, \\
W(k)=0 \text { for any } k \in \mathbb{Z}, \\
W>0 \text { in } \mathbb{R} \backslash \mathbb{Z}, \\
W^{\prime \prime}(0)>0 .
\end{array}\right.
$$

As customary, $\epsilon>0$ is a small scale parameter, and $\sigma_{\epsilon}$ plays the role of an exterior stress acting on the material. We suppose that

$$
\sigma_{\epsilon}(t, x):=\epsilon^{2 s} \sigma\left(\epsilon^{1+2 s} t, \epsilon x\right),
$$

where $\sigma$ is a bounded uniformly continuous function such that, for some $\alpha \in(s, 1)$ and $M>0$, it holds

$$
\begin{aligned}
& \left\|\sigma_{x}\right\|_{L^{\infty}([0,+\infty) \times \mathbb{R})}+\left\|\sigma_{t}\right\|_{L^{\infty}([0,+\infty) \times \mathbb{R})} \leqslant M, \\
& \left|\sigma_{x}(t, x+h)-\sigma_{x}(t, x)\right| \leqslant M|h|^{\alpha}, \quad \text { for every } x, h \in \mathbb{R} \text { and } t \in[0,+\infty) .
\end{aligned}
$$

The problem in (1.1) arises in the classical Peierls-Nabarro model for atomic dislocation in crystals, see e.g. [15] and references therein. We will recall the basics of such model in the subsequent Section 2. Now, setting

$$
v_{\epsilon}(t, x):=v\left(\frac{t}{\epsilon^{1+2 s}}, \frac{x}{\epsilon}\right),
$$

we look at the equation satisfied by the rescaled function $v_{\epsilon}$, that is, recalling (1.1),

$$
\left\{\begin{array}{l}
\left(v_{\epsilon}\right)_{t}=\frac{1}{\epsilon}\left(L_{s} v_{\epsilon}-\frac{1}{\epsilon^{2 s}} W^{\prime}\left(v_{\epsilon}\right)+\sigma(t, x)\right) \quad \text { in }(0,+\infty) \times \mathbb{R}, \\
v_{\epsilon}(0, \cdot)=v_{\epsilon}^{0} \quad \text { in } \mathbb{R} .
\end{array}\right.
$$

To suitably choose the initial condition $v_{\epsilon}^{0}$ and to state our main result, we introduce the basic layer solution $u$, that is the solution of the problem

$$
\left\{\begin{array}{l}
L_{s} u-W^{\prime}(u)=0 \quad \text { in } \mathbb{R}, \\
u^{\prime}>0 \text { and } u(-\infty)=0, \quad u(0)=1 / 2, \quad u(+\infty)=1 .
\end{array}\right.
$$

For the existence of such solution and its main properties see $[19,5]$. An interesting feature of this solution is the fact that it approaches its limits at $\pm \infty$ with a polynomial decay, namely

$$
\left|u(x)-H(x)+\frac{1}{2 s W^{\prime \prime}(0)} \frac{x}{|x|^{1+2 s}}\right| \leqslant \frac{C}{|x|^{1+2 s}} \quad \text { for any } x \in \mathbb{R},
$$

as we will show in Proposition 7.2 (here and in the rest of the paper, $H$ is the Heaviside $^{1}$ step function). When $s=1 / 2$ the estimate in (1.6) is somehow simpler, thanks to some explicit representation formulas: in our case, to prove (1.6) we

\footnotetext{
${ }^{1}$ Namely, $H(x)=0$ if $x<0, H(x)=1$ if $x>0$ (the explicit value at $x=0$, that is assumed to be in $[0,1]$, plays no role).
} 
will construct by hand a particular solution for a different potential and scale it appropriately in order to fit the asymptotics of the original solution.

We recall that the semi-continuous envelopes of $u$ are defined as

$$
u^{*}(t, x)=\limsup _{\left(t^{\prime}, x^{\prime}\right) \rightarrow(t, x)} u\left(t^{\prime}, x^{\prime}\right)
$$

and

$$
u_{*}(t, x)=\liminf _{\left(t^{\prime}, x^{\prime}\right) \rightarrow(t, x)} u\left(t^{\prime}, x^{\prime}\right) .
$$

Moreover, given $x_{1}^{0}<x_{2}^{0}<\ldots<x_{N}^{0}$, we consider the solution $\left(x_{i}(t)\right)_{i=1, \ldots, N}$ to the system

$$
\left\{\begin{array}{l}
\dot{x_{i}}=\gamma\left(-\sigma\left(t, x_{i}\right)+\sum_{j \neq i} \frac{x_{i}-x_{j}}{2 s\left|x_{i}-x_{j}\right|^{2 s+1}}\right) \text { in }(0,+\infty), \\
x_{i}(0)=x_{i}^{0}
\end{array}\right.
$$

where

$$
\gamma=\left(\int_{\mathbb{R}}\left(u^{\prime}\right)^{2}\right)^{-1}
$$

For the existence and uniqueness of such solution see Section 8 in [9]. We consider as initial condition in (1.4) the state

$$
v_{\epsilon}^{0}(x)=\frac{\epsilon^{2 s}}{\beta} \sigma(0, x)+\sum_{i=1}^{N} u\left(\frac{x-x_{i}^{0}}{\epsilon}\right),
$$

where

$$
\beta:=W^{\prime \prime}(0)>0 .
$$

The main result obtained in this framework is the following:

Theorem 1.1. Assume that assumptions (1.2), (1.3) and (1.10) hold, and let

$$
v_{0}(t, x)=\sum_{i=1}^{N} H\left(x-x_{i}(t)\right)
$$

where $H$ is the Heaviside function, and $\left(x_{i}(t)\right)_{i=1, \ldots, N}$ is the solution to (1.8).

Then, for every $\epsilon>0$ there exists a unique viscosity solution $v_{\epsilon}$ to (1.4). Furthermore, as $\epsilon \rightarrow 0$, the solution $v_{\epsilon}$ exhibits the following asymptotic behavior:

$$
\limsup _{\substack{\left(t^{\prime}, x^{\prime}\right) \rightarrow(t, x) \\ \epsilon \rightarrow 0}} v_{\epsilon}\left(t^{\prime}, x^{\prime}\right) \leqslant\left(v_{0}\right)^{*}(t, x)
$$

and

$$
\liminf _{\substack{\left(t^{\prime}, x^{\prime}\right) \rightarrow(t, x) \\ \epsilon \rightarrow 0}} v_{\epsilon}\left(t^{\prime}, x^{\prime}\right) \geqslant\left(v_{0}\right)_{*}(t, x)
$$

for any $t \in[0,+\infty)$ and $x \in \mathbb{R}$. 
When $s=1 / 2$ the result above was proved in [11], where it was also raised the question about what happens for other values of the parameter $s$.

It is worth pointing out that one of the crucial ingredients used in [11] (see, in particular, pages 20-21 there) is the precise knowledge of the asymptotics of the layer solutions in the case when $s=1 / 2$. In [11], this was obtained by passing through the harmonic extension, in order to use a comparison argument with the solution of the Peierls-Nabarro problem, which has an explicit representation when $s=1 / 2$. On the contrary, in the arguments we provide here, we follow a different approach that avoids both the harmonic extension (that is somehow specialized for fractional Laplacian operators but does not fit other more general equations) and the use of explicit formulas for solutions (that are only available when $s=1 / 2$ ). For us, the improved asymptotics for the layer solutions $u$ of (1.5) is based on an auxiliary class of potentials $V$ coupled with the solutions $\phi$ of the corresponding problems $L_{s} \phi=V^{\prime}(\phi)$, which we are able to control via the construction of suitable barriers. Then, in a subsequent step, this general approach will permit us to provide a precise order of approximation also for the asymptotics of the original layer solutions $u$ in terms of the original potential $W$.

Since, differently from [11], we do not make use of any harmonic extension results, that are specific for the fractional powers of the Laplacian, our proof is feasible for more general types of integro-differential equations (as a matter of fact, our approach provides a different proof also for the case $s=1 / 2$ treated in [11]).

In the course of the proof, our basic strategy is to make the ansatz that the solution is, at the first order, the superposition of transition layers. To make this rigorous, a suitable corrector needs to be introduced in order to take care of the higher order remainders.

The rest of the paper is organized as follows. In Section 2, we will give a quick review of the Peierls-Nabarro model, giving a physical interpretation to the results in Theorem 1.1. In Section 3, we will present some formal heuristics on the transition layers, in order to explain how the ODE system in (1.8) naturally appears as the leading order of the dislocation evolution.

Then, the rigorous mathematical treatment of the problem begins in Section 4, where we point out a comparison principle that will be used in Section 5 to introduce the layer solution and the solution to an auxiliary corrector equation, needed to approximate the solution at a sufficiently high order. In Section 6 , we construct an auxiliary transition layer driven by a suitable potential, which will be used in Section 7 to control the standard transition layer up to the desired order of approximation. The proof of Theorem 1.1 will be completed in Section 8 .

\section{Physical motivations}

We recall here a simple (and even oversimplified) version of a model for the dynamics of the dislocation of atoms in crystals. The model is related to the Peierls-Nabarro energy functional that combines the elastic properties of the material with a misfit occurring along a glide line (for simplicity, we consider here a two-dimensional model). The system is in fact a hybrid combination in which 
a discrete dislocation occurring along a slide line is incorporated in a continuum medium. Of course, any hybrid system is based somehow on a radical approximation of the structure of the matter, but this may also lead to equations that are more convenient to deal with analytically than the ones arising in more detailed, but less treatable, models. Also, in spite of the simplicity of the equations obtained, the Peierls-Nabarro model and its modifications are commonly considered as good explanations for the plastic behaviors of some metals.

In our mathematical setting, the medium will be the plane $\mathbb{R}^{2}$, endowed with coordinates $(x, y)$, and the glide line will be taken as the $x$-axis. As a matter of fact, since the argument is symmetric, we will focus only on the energy contribution of the upper half-plane $\mathbb{R}_{+}^{2}:=\left\{(x, y) \in \mathbb{R}^{2}\right.$ s.t. $\left.y \geqslant 0\right\}$ (or, simply, we make a slide deformation in the upper part of the crystal, by keeping the lower part fixed).

We suppose that the material has a crystalline structure that leads atoms to display periodically. Namely, the atoms on the $x$-axis have the preference of occupying the integer sites, in virtue of the configuration of the crystal on a large scale. If a misfit occurs (e.g. due to an external stress) which moves some atoms out of their rest position, the material will react trying to restore a crystalline configuration, that is either the original configuration or one obtained by translating the configuration by the natural periodicity of the crystal. This effect may be modeled by defining $v^{0}(x)$ to be the discrepancy between the position of the atom $x$ with its rest position (hence, at a global scale, the misfit of the crystal on the glide line is $\left.v^{0}(+\infty)-v^{0}(-\infty)\right)$. Then, the misfit energy is taken as

$$
\mathscr{M}\left(v^{0}\right):=\int_{\mathbb{R}} W\left(v^{0}(x)\right) \mathrm{d} x .
$$

Notice that (1.2) assures that $\mathscr{M}$ is minimized when all the atoms are at rest (i. e., $v \equiv 0$ ) as well as when all the atoms are shifted to another periodic configurations (e.g., $v \equiv 1$ ). 


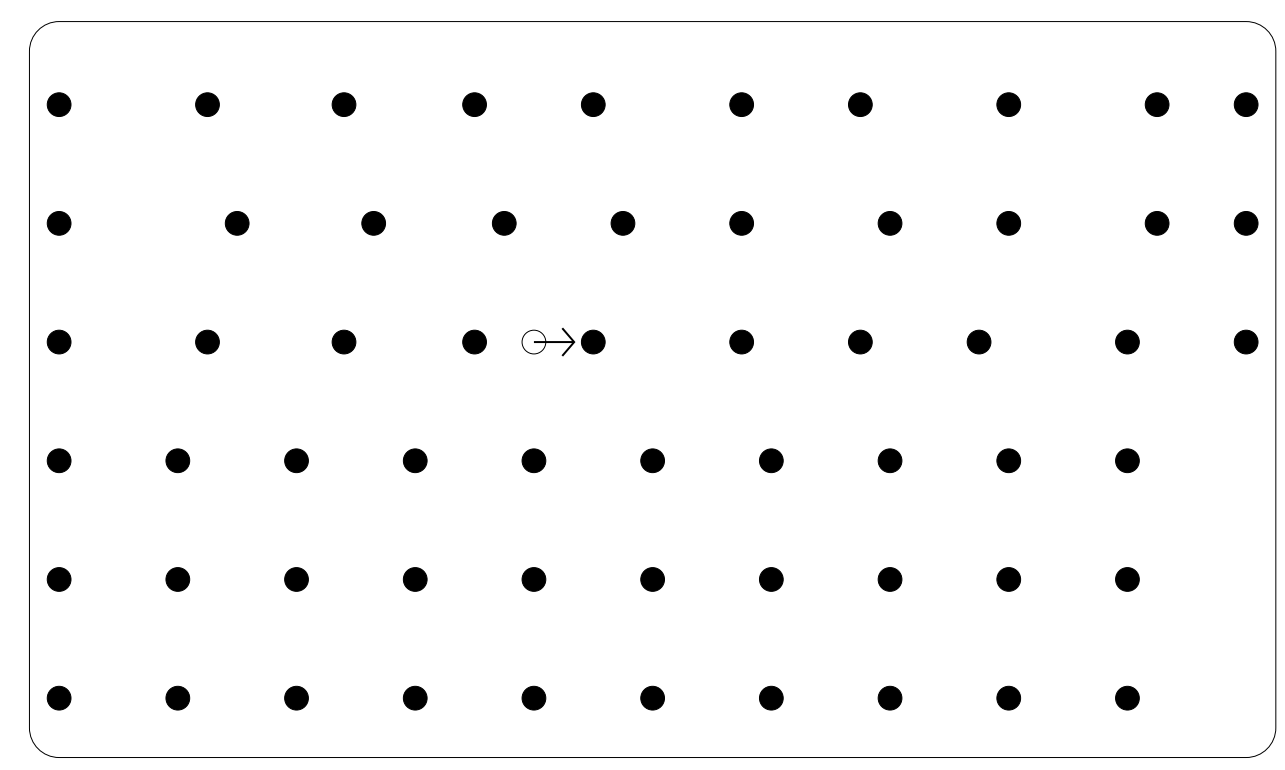

Figure 1

The material ${ }^{2}$ is also influenced by the classical elastic energy. That is, by considering the dislocation function $v(x, y)$ on all $\mathbb{R}_{+}^{2}$ (notice that $v(x, 0)=v^{0}(x)$ ), the elastic contribution is given by

$$
\mathscr{E}(v):=\frac{1}{2} \int_{\mathbb{R}_{+}^{2}}|\nabla v(x, y)|^{2} \mathrm{~d} x \mathrm{~d} y .
$$

The total energy of the system is, therefore,

$$
\mathscr{F}(v):=\mathscr{E}(v)+\mathscr{M}\left(v^{0}\right)=\frac{1}{2} \int_{\mathbb{R}_{+}^{2}}|\nabla v(x, y)|^{2} \mathrm{~d} x \mathrm{~d} y+\int_{\mathbb{R}} W(v(x, 0)) \mathrm{d} x,
$$

up to dimensional constants that we neglect. We remark that critical points of $\mathscr{F}$ (and in particular energy minimizers) satisfy the equation

$$
\left\{\begin{array}{cc}
\Delta v(x, y)=0 & \text { for any } x \in \mathbb{R} \text { and } y>0, \\
\partial_{y} v(x, 0)=W^{\prime}(v(x, 0)) & \text { for any } x \in \mathbb{R}
\end{array}\right.
$$

that is, up to a normalizing constant, $L_{1 / 2} v^{0}(x)=W^{\prime}\left(v^{0}(x)\right)$ for any $x \in \mathbb{R}$ (to be compared with (1.5)). The corresponding parabolic evolution equation is $v_{t}^{0}=L_{1 / 2} v^{0}-W^{\prime}\left(v^{0}\right)$ (to be compared with the forced analogue in (1.1)). The operator $L_{s}$ (and even more general types of operators) may be obtained with this construction in presence of heterogeneous media.

\footnotetext{
${ }^{2}$ Figure 1 is an attempt of describing the deformation in a portion of the crystal. Though the crystal is infinite, each row drawn in the picture contains ten atoms, for simplicity. The three upper rows belong to $\mathbb{R}_{+}^{2}$, while the three rows on the bottom lie in $\mathbb{R}^{2} \backslash \mathbb{R}_{+}^{2}$. The arrow on the third row represents $v^{0}$, that is the dislocation from the original position of the atom, which is represented by a void circle: in this case, if the period of the crystal is 1 , then $v^{0}$ there is approximatively 0.5 , while $v^{0}$ on the extreme left (respectively, on the extreme right) is approximatively 0 (respectively, 1 ).
} 
As a matter of fact, we can perform a small variation of the model above to obtain the equation for $v^{0}$ by an argument which only involves the elastic reactions on the slip line. For this, we suppose that the displacement $v^{0}$ causes an elastic reaction on the slip plane with some polynomial decay. For instance, suppose that the elastic energy density at the point $x$ of the slip line is proportional to $v^{0}(x)$, say

$$
d \mathscr{E}^{0}\left(v^{0} ; x\right)=\mathscr{K}(x) v^{0}(x) \mathrm{d} x
$$

and assume that $\mathscr{K}(x)$ arises from the variation of $v^{0}$ along the line, weighted by a polynomially decreasing tail, such as, in the principal value sense,

$$
\mathscr{K}(x)=\int_{\mathbb{R}} \frac{v^{0}(x)-v^{0}(y)}{|x-y|^{1+2 s}} \mathrm{~d} y .
$$

Then, at least formally, we have that

$$
\begin{aligned}
\mathscr{E}^{0}\left(v^{0}\right)= & \int_{\mathbb{R}} d \mathscr{E}^{0}\left(v^{0} ; x\right) \\
= & \int_{\mathbb{R}} \int_{\mathbb{R}} \frac{\left(v^{0}(x)-v^{0}(y)\right) v^{0}(x)}{|x-y|^{1+2 s}} \mathrm{~d} x \mathrm{~d} y \\
= & \frac{1}{2} \int_{\mathbb{R}} \int_{\mathbb{R}} \frac{\left(v^{0}(x)\right)^{2}}{|x-y|^{1+2 s}} \mathrm{~d} x \mathrm{~d} y+\frac{1}{2} \int_{\mathbb{R}} \int_{\mathbb{R}} \frac{\left(v^{0}(y)\right)^{2}}{|x-y|^{1+2 s}} \mathrm{~d} x \mathrm{~d} y \\
& \quad-\int_{\mathbb{R}} \int_{\mathbb{R}} \frac{v^{0}(y) v^{0}(x)}{|x-y|^{1+2 s}} \mathrm{~d} x \mathrm{~d} y \\
= & \frac{1}{2} \int_{\mathbb{R}} \int_{\mathbb{R}} \frac{\left(v^{0}(x)-v^{0}(y)\right)^{2}}{|x-y|^{1+2 s}} \mathrm{~d} x \mathrm{~d} y .
\end{aligned}
$$

In this framework, the total energy becomes

$$
\mathscr{E}^{0}\left(v^{0}\right)+\mathscr{M}\left(v^{0}\right)=\frac{1}{2} \int_{\mathbb{R}} \int_{\mathbb{R}} \frac{\left(v^{0}(x)-v^{0}(y)\right)^{2}}{|x-y|^{1+2 s}} \mathrm{~d} x \mathrm{~d} y+\int_{\mathbb{R}} W(v(x, 0)) \mathrm{d} x,
$$

up to dimensional constants, and critical points of this energy functional satisfy $L_{s} v^{0}(x)=W^{\prime}\left(v^{0}(x)\right)$ (compare again with (1.5) and (1.1)).

In these physical models, Theorem 1.1 studies the (sufficiently long) time evolution in a crystal with (sufficiently small) periodic scale of the atom dislocation under an external stress. The initial configuration taken into account in (1.10) corresponds, roughly and in the appropriate space and time scaling, to $N$ separate dislocations that are as intense as one single period of the crystal and are centered at $x_{1}^{0}, \ldots, x_{N}^{0}$, possibly plus an external strain deformation. This dislocation function evolves in time approaching the superposition of $N$ pure deformations, each of the size of the periodicity of the crystal: in this sense, at least at a macroscopic space and time scale, dislocations occur at single points of the crystal, and each dislocation has exactly the same size as the periodicity of the crystal (see (1.12); the envelopes in (1.13) and (1.14) are mainly needed to obtain a semi-continuous interpolation of the profile from both sides, thus resolving the ambiguity of the notion of the displacement function at its jump points). 
These pure deformations are centered at points $x_{1}(t), \ldots, x_{N}(t)$ that evolve according to (1.8): that is, they react elastically against the external stress ${ }^{3}$ and interact one with the other in a repulsive way.

In this sense, Theorem 1.1 gives a detailed and rigorous mathematical description of the creation, displacement and motion of defects in crystals under a small external stress.

Models similar to the one discussed here were presented also in $[1,2,6,7,10$, $12,13,14,17,18,23,24]$. For further discussions about the dislocation dynamics also in relation with homogenization properties see e.g. [16] and references therein.

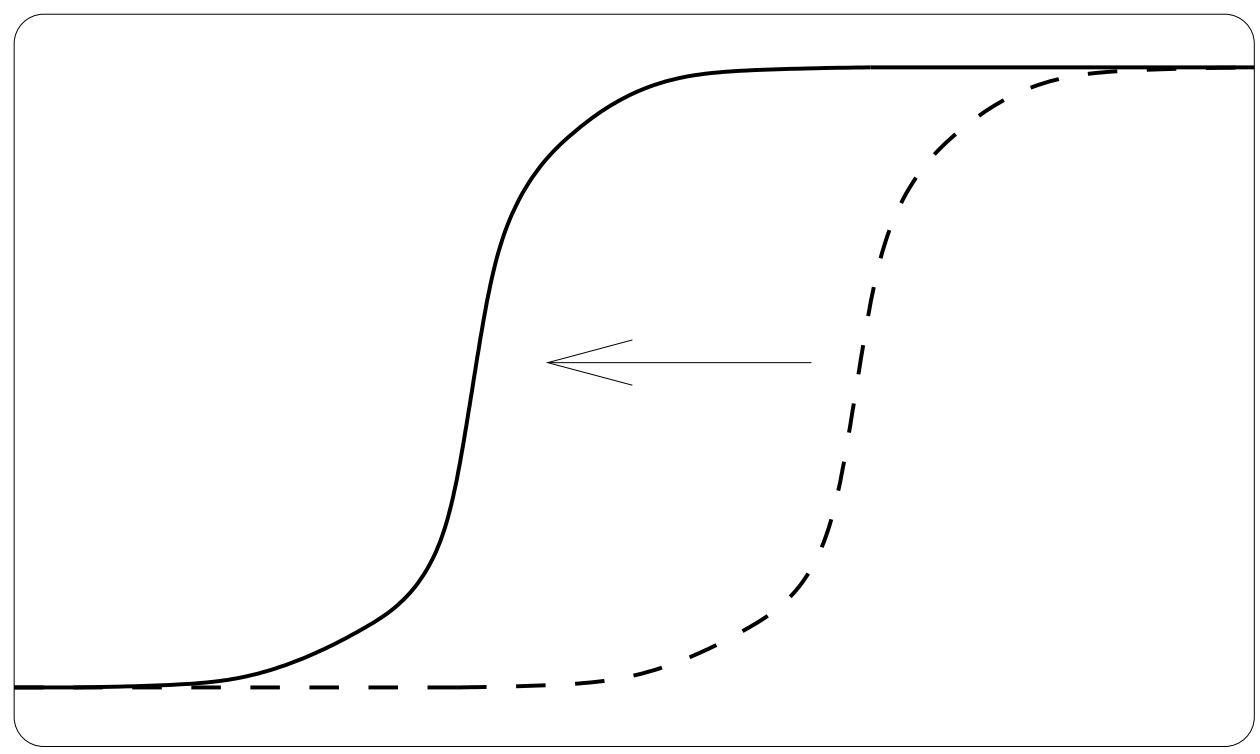

Figure 2

\section{Heuristics ON THE DYNAMICS OF THE TRANSITION LAYERS}

Here we would like to give a formal (absolutely not rigorous) justification of the ODE system in (1.8) that drives the motion of the transition layers.

\footnotetext{
${ }^{3}$ Notice a sign change in the stress between (1.1) and (1.8). There is no mystery in this sign change, as we try to explain in Figure 2, where a single translation is considered in case of a positive stress $\sigma$. Suppose that the dashed curve represents the transition $v$ at time $t$. Then, at any given point, the positive stress will try to increase the value of $v$ at time $t+\delta t$, according to (1.1). The result of this increasing the value of the function is represented by the solid curve in Figure 2. All in all, the dashed transition at time $t$ has moved towards the left to the solid transition at time $t+\delta t$. This clearly suggests that a positive stress results into a motion of the transition points towards the negative side and explains the sign change for $\sigma$ from (1.1) to (1.8).
} 
For this, we use the notation $\simeq$ to denote equality up to negligible terms in $\epsilon$ and we exploit the notation

$$
u_{\epsilon, i}(t, x):=u\left(\frac{x-x_{i}(t)}{\epsilon}\right) .
$$

With a slight abuse of notation we write

$$
u_{\epsilon, i}^{\prime}(t, x):=u^{\prime}\left(\frac{x-x_{i}(t)}{\epsilon}\right) .
$$

We also write $\nu$ for a generic integer number: e. g., we will write that the Heaviside step function $H$ is equal to $\nu$, since it is equal to either 0 or 1 , and notice that $W^{\prime}(\nu)=0$ and $W(r+\nu)=W(r)$ for any $r \in \mathbb{R}$. In this setting, by (1.6), we have that

$$
\begin{aligned}
u_{\epsilon, i}(t, x) & \simeq H\left(\frac{x-x_{i}}{\epsilon}\right)-\frac{\epsilon^{2 s}\left(x-x_{i}\right)}{2 s W^{\prime \prime}(0)\left|x-x_{i}\right|^{1+2 s}} \\
& =\nu-\frac{\epsilon^{2 s}\left(x-x_{i}\right)}{2 s W^{\prime \prime}(0)\left|x-x_{i}\right|^{1+2 s}} .
\end{aligned}
$$

The idea is now to use the ansatz that the solution $v_{\epsilon}$ is well-approximated by the sum of $N$ transitions, and plug this expression into equation (1.4). For this we write

$$
v_{\epsilon}(t, x) \simeq \sum_{i=1}^{N} u_{\epsilon, i}(t, x)=\sum_{i=1}^{N} u\left(\frac{x-x_{i}(t)}{\epsilon}\right)
$$

so that

$$
\text { and } \begin{aligned}
\left(v_{\epsilon}\right)_{t} & =-\sum_{i=1}^{N} u^{\prime}\left(\frac{x-x_{i}(t)}{\epsilon}\right) \frac{\dot{x}_{i}(t)}{\epsilon} \\
L_{s} v_{\epsilon} & =\frac{1}{\epsilon^{2 s}} \sum_{i=1}^{N}\left(L_{s} u\right)\left(\frac{x-x_{i}(t)}{\epsilon}\right) \\
& =\frac{1}{\epsilon^{2 s}} \sum_{i=1}^{N} W^{\prime}\left(u\left(\frac{x-x_{i}(t)}{\epsilon}\right)\right) \\
& =\frac{1}{\epsilon^{2 s}} \sum_{i=1}^{N} W^{\prime}\left(u_{\epsilon, i}(t, x)\right) .
\end{aligned}
$$

By inserting into (1.4), after a multiplication by $\epsilon$ we obtain

$$
\begin{aligned}
-\sum_{i=1}^{N} u^{\prime} & \left(\frac{x-x_{i}(t)}{\epsilon}\right) \dot{x}_{i}(t) \\
& =\frac{1}{\epsilon^{2 s}}\left[\sum_{i=1}^{N} W^{\prime}\left(u_{\epsilon, i}(t, x)\right)-W^{\prime}\left(\sum_{i=1}^{N} u_{\epsilon, i}(t, x)\right)+\epsilon^{2 s} \sigma(t, x)\right] .
\end{aligned}
$$

Now we make some observations on the asymptotics of the potential $W$. First of all, we notice that

$$
\int_{\mathbb{R}} W^{\prime}(u(x)) u^{\prime}(x) \mathrm{d} x=\int_{\mathbb{R}} \frac{\mathrm{d}}{\mathrm{d} x} W(u(x)) \mathrm{d} x=W(1)-W(0)=0 .
$$


Thus, by changing variable,

$$
\int_{\mathbb{R}} W^{\prime}\left(u_{\epsilon, k}(t, x)\right) u_{\epsilon, k}^{\prime}(t, x) \mathrm{d} x=0 .
$$

Also, we observe that, if $i \neq k$, the function

$$
y \mapsto u\left(y+\frac{x_{k}-x_{i}}{\epsilon}\right)
$$

is asymptotic to either 0 (if $x_{k}<x_{i}$ ) or 1 (if $x_{k}>x_{i}$ ) for small $\epsilon$, hence we write

$$
u\left(y+\frac{x_{k}-x_{i}}{\epsilon}\right) \simeq \nu
$$

for any $i \neq k$. Thus,

$$
\begin{aligned}
W^{\prime}\left(\sum_{i=1}^{N} u\left(y+\frac{x_{k}-x_{i}}{\epsilon}\right)\right) & =W^{\prime}\left(u(y)+\sum_{\substack{1 \leq i \leq N \\
i \neq k}} u\left(y+\frac{x_{k}-x_{i}}{\epsilon}\right)\right) \\
& \simeq W^{\prime}(u(y)+\nu)=W^{\prime}(u(y)) .
\end{aligned}
$$

So, we use the substitution $y:=\left(x-x_{k}\right) / \epsilon$ to see that

$$
\begin{aligned}
\frac{1}{\epsilon} \int_{\mathbb{R}} W^{\prime}\left(\sum_{i=1}^{N} u_{\epsilon, i}(t, x)\right) u_{\epsilon, k}^{\prime}(t, x) \mathrm{d} x \\
\quad=\int_{\mathbb{R}} W^{\prime}\left(\sum_{i=1}^{N} u\left(y+\frac{x_{k}-x_{i}}{\epsilon}\right)\right) u^{\prime}(y) \mathrm{d} y \\
\simeq \int_{\mathbb{R}} W^{\prime}(u(y)) u^{\prime}(y) \mathrm{d} y=0,
\end{aligned}
$$

thanks to (3.3). Using (3.5) and (3.4), we obtain that

$$
\begin{aligned}
\frac{1}{\epsilon^{2 s}} \int_{\mathbb{R}}\left[\sum_{i=1}^{N} W^{\prime}\left(u_{\epsilon, i}(t, x)\right)-W^{\prime}\left(\sum_{i=1}^{N} u_{\epsilon, i}(t, x)\right)+\epsilon^{2 s} \sigma(t, x)\right] u_{\epsilon, k}^{\prime}(t, x) \mathrm{d} x \\
\simeq \frac{1}{\epsilon^{2 s}} \int_{\mathbb{R}}\left[\sum_{i=1}^{N} W^{\prime}\left(u_{\epsilon, i}(t, x)\right)+\epsilon^{2 s} \sigma(t, x)\right] u_{\epsilon, k}^{\prime}(t, x) \mathrm{d} x \\
=\frac{1}{\epsilon^{2 s}} \int_{\mathbb{R}}\left[\sum_{\substack{1 \leqslant i \leqslant N \\
i \neq k}} W^{\prime}\left(u_{\epsilon, i}(t, x)\right)+\epsilon^{2 s} \sigma(t, x)\right] u_{\epsilon, k}^{\prime}(t, x) \mathrm{d} x
\end{aligned}
$$


Now we use (3.1), the fact that $W^{\prime}(0)=0$ and a Taylor expansion to see that

$$
\begin{aligned}
W^{\prime}\left(u_{\epsilon, i}(t, x)\right) & \simeq W^{\prime}\left(\nu-\frac{\epsilon^{2 s}\left(x-x_{i}\right)}{2 s W^{\prime \prime}(0)\left|x-x_{i}\right|^{1+2 s}}\right) \\
& =W^{\prime}\left(\frac{-\epsilon^{2 s}\left(x-x_{i}\right)}{2 s W^{\prime \prime}(0)\left|x-x_{i}\right|^{1+2 s}}\right) \\
& \simeq W^{\prime \prime}(0) \cdot \frac{-\epsilon^{2 s}\left(x-x_{i}\right)}{2 s W^{\prime \prime}(0)\left|x-x_{i}\right|^{1+2 s}} \\
& =\frac{-\epsilon^{2 s}\left(x-x_{i}\right)}{2 s\left|x-x_{i}\right|^{1+2 s}} .
\end{aligned}
$$

Therefore, using the substitution $y:=\left(x-x_{k}\right) / \epsilon$, we obtain that, for any $i \neq k$,

$$
\begin{aligned}
\frac{1}{\epsilon} \int_{\mathbb{R}} W^{\prime}\left(u_{\epsilon, i}(t, x)\right) u_{\epsilon, k}^{\prime}(t, x) \mathrm{d} x & \simeq-\frac{1}{\epsilon} \int_{\mathbb{R}} \frac{\epsilon^{2 s}\left(x-x_{i}\right)}{2 s\left|x-x_{i}\right|^{1+2 s}} u^{\prime}\left(\frac{x-x_{k}}{\epsilon}\right) \mathrm{d} x \\
& =-\int_{\mathbb{R}} \frac{\epsilon^{2 s}\left(x_{k}-x_{i}+\epsilon y\right)}{2 s\left|x_{k}-x_{i}+\epsilon y\right|^{1+2 s}} u^{\prime}(y) \mathrm{d} y \\
& \simeq-\int_{\mathbb{R}} \frac{\epsilon^{2 s}\left(x_{k}-x_{i}\right)}{2 s\left|x_{k}-x_{i}\right|^{1+2 s}} u^{\prime}(y) \mathrm{d} y \\
& =\frac{-\epsilon^{2 s}\left(x_{k}-x_{i}\right)}{2 s\left|x_{k}-x_{i}\right|^{1+2 s}} .
\end{aligned}
$$

Moreover

$$
\begin{aligned}
\frac{1}{\epsilon} \int_{\mathbb{R}} \sigma(t, x) u_{\epsilon, k}^{\prime}(t, x) \mathrm{d} x & =\int_{\mathbb{R}} \sigma\left(t, x_{k}+\epsilon y\right) u^{\prime}(y) \mathrm{d} y \\
& \simeq \int_{\mathbb{R}} \sigma\left(t, x_{k}\right) u^{\prime}(y) \mathrm{d} y=\sigma\left(t, x_{k}\right) .
\end{aligned}
$$

So we plug (3.7) and (3.8) into (3.6) and we conclude that

$$
\begin{gathered}
\frac{1}{\epsilon^{2 s}} \int_{\mathbb{R}}\left[\sum_{i=1}^{N} W^{\prime}\left(u_{\epsilon, i}(t, x)\right)-W^{\prime}\left(\sum_{i=1}^{N} u_{\epsilon, i}(t, x)\right)+\epsilon^{2 s} \sigma(t, x)\right] u_{\epsilon, k}^{\prime}(t, x) \mathrm{d} x \\
\simeq-\sum_{\substack{1 \leqslant i \leqslant N \\
i \neq k}} \frac{\epsilon\left(x_{k}-x_{i}\right)}{2 s\left|x_{k}-x_{i}\right|^{1+2 s}}+\epsilon \sigma\left(t, x_{k}\right) .
\end{gathered}
$$

Observe now that, if $i \neq k$, the function

$$
y \mapsto u^{\prime}\left(y+\frac{x_{k}-x_{i}}{\epsilon}\right)
$$

is asymptotic to $u^{\prime}( \pm \infty)=0$ for small $\epsilon$, therefore

$$
\begin{aligned}
\int_{\mathbb{R}} u^{\prime}\left(\frac{x-x_{i}}{\epsilon}\right) & u_{\epsilon, k}^{\prime}(t, x) \mathrm{d} x \\
& =\epsilon \int_{\mathbb{R}} u^{\prime}\left(y+\frac{x_{k}-x_{i}}{\epsilon}\right) u^{\prime}(y) \mathrm{d} y \simeq 0
\end{aligned}
$$

if $i \neq k$. Similarly,

$$
\int_{\mathbb{R}} u^{\prime}\left(\frac{x-x_{k}}{\epsilon}\right) u_{\epsilon, k}^{\prime}(t, x) \mathrm{d} x=\epsilon \int_{\mathbb{R}}\left(u^{\prime}(y)\right)^{2} \mathrm{~d} y=\frac{\epsilon}{\gamma},
$$


by (1.9). Now we test (3.2) against $u_{\epsilon, k}^{\prime}$ and we recall (3.9). We obtain

$$
-\epsilon \gamma^{-1} \dot{x}_{k}(t)=-\sum_{\substack{1 \leqslant i \leqslant N \\ i \neq k}} \frac{\epsilon\left(x_{k}-x_{i}\right)}{2 s\left|x_{k}-x_{i}\right|^{1+2 s}}+\epsilon \sigma\left(t, x_{k}\right),
$$

which gives (1.8) after a division by $\epsilon$.

\section{A COMPARISON PRINCIPLE}

For further use, we present here a comparison principle for sub and supersolutions decaying at infinity:

Proposition 4.1. Let $v \in L^{\infty}(\mathbb{R}) \cap C^{2}(\mathbb{R})$ such that

$$
\lim _{x \rightarrow \pm \infty} v(x)=0 .
$$

Suppose that there exists a function $d \in L^{\infty}(\mathbb{R})$ such that $d(x) \geqslant 0$ for any $x \in \mathbb{R}$, and $L_{s} v \geqslant d v$ (respectively $L_{s} v \leqslant d v$ ). Then either $v \equiv 0$ or $v<0$ (respectively $v>$ $0)$.

Proof. We prove first that

$$
L_{s} v \geqslant d v \Longrightarrow v \leqslant 0 .
$$

By contradiction, assume that the function $v$ reaches its maximum at a point $\bar{x}$ such that $v(\bar{x})>0$. Therefore,

$$
L_{s} v(\bar{x})=\frac{1}{2} \int_{\mathbb{R}} \frac{v(\bar{x}+y)+v(\bar{x}-y)-2 v(\bar{x})}{|y|^{1+2 s}} \mathrm{~d} y \leqslant 0
$$

since $v(x) \leqslant v(\bar{x})$ for any $x \in \mathbb{R}$. On the other hand,

$$
L_{s} v(\bar{x}) \geqslant d v(\bar{x}) \geqslant 0 .
$$

From (4.3) and (4.4) we get that

$$
v(\bar{x}+y)+v(\bar{x}-y)-2 v(\bar{x})=0
$$

for any $y \in \mathbb{R}$, hence $v$ is constantly equal to its maximal value $v(\bar{x})$. From the fact that such value is strictly positive, we reach a contradiction with (4.1). This proves (4.2).

Now, we want to show that $v<0$ unless $v \equiv 0$. For this, we suppose that $v \not \equiv 0$ and that there exists a point $\tilde{x}$ such that $v(\tilde{x})=0 \geqslant v(x)$ for any $x \in \mathbb{R}$, with strict inequality in a non empty domain. Hence,

$$
L_{s} v(\tilde{x})=\frac{1}{2} \int_{\mathbb{R}} \frac{v(\tilde{x}+y)+v(\tilde{x}-y)-2 v(\tilde{x})}{|y|^{1+2 s}} \mathrm{~d} y<0
$$

and

$$
L_{s} v(\tilde{x}) \geqslant d v(\tilde{x})=0 .
$$

The inequalities above give a contradiction. This concludes the proof of the proposition. 


\section{LAYER SOLUTION AND CORRECTOR}

We recall the following layer solution construction given in Theorem 2 of [19]:

Theorem 5.1. Under the assumptions on the potential $W$ given in (1.2), there exists a unique solution $u$ to (1.5). Moreover, $u \in C^{2}(\mathbb{R})$ and there exists a constant $C \geqslant 1$ such that

$$
|u(x)-H(x)| \leqslant C|x|^{-2 s} \quad \text { and } \quad\left|u^{\prime}(x)\right| \leqslant C|x|^{-(1+2 s)}
$$

for any large $x \in \mathbb{R}$.

As for the corrector equation, we consider the following problem:

$$
\left\{\begin{array}{l}
L_{s} \psi-W^{\prime \prime}(u) \psi=u^{\prime}+\eta\left(W^{\prime \prime}(u)-W^{\prime \prime}(0)\right) \text { in } \mathbb{R}, \\
\psi(-\infty)=0=\psi(+\infty)
\end{array}\right.
$$

where

$$
\eta=\frac{\int_{\mathbb{R}}\left(u^{\prime}(x)\right)^{2} \mathrm{~d} x}{W^{\prime \prime}(0)} .
$$

The fact that (5.2) is a good ansatz for the corrector equation may be heuristically inferred from the computations in Section 3.1 of [11] (see, in particular, formula (3.18) there). The existence of a solution for such problem is given by the following

Theorem 5.2. Suppose that the assumptions on the potential $W$ in (1.2) hold. Then, there exists a unique solution $\psi \in H^{s}(\mathbb{R})$ to (5.2). Furthermore, we have that $\psi \in C_{\text {loc }}^{1, \alpha}(\mathbb{R}) \cap L^{\infty}(\mathbb{R})$, for some $\alpha \in(0,1)$, and

$$
\left\|\psi^{\prime}\right\|_{L^{\infty}(\mathbb{R})}<+\infty \text {. }
$$

For the proof of Theorem 5.2, we consider the following bilinear form on $H^{s}(\mathbb{R})$

$$
\mathscr{L}(v, w):=\int_{\mathbb{R}} \int_{\mathbb{R}} \frac{(v(x)-v(y))(w(x)-w(y))}{|x-y|^{1+2 s}} \mathrm{~d} x \mathrm{~d} y+\int_{\mathbb{R}} W^{\prime \prime}(u) v w \mathrm{~d} x,
$$

and the quadratic functional

$$
\mathscr{G}(v):=\frac{1}{2} \int_{\mathbb{R}} \int_{\mathbb{R}} \frac{|v(x)-v(y)|^{2}}{|x-y|^{1+2 s}} \mathrm{~d} x \mathrm{~d} y+\frac{1}{2} \int_{\mathbb{R}} W^{\prime \prime}(u) v^{2} \mathrm{~d} x,
$$

where $u$ is the layer solution introduced in (1.5). Also, we consider the closed subset $\mathcal{X} \subset H^{s}(\mathbb{R})$ given by the functions $v$ orthogonal to $u^{\prime}$ in $L^{2}(\mathbb{R})$; that is,

$$
\mathcal{X}:=\left\{v \in H^{s}(\mathbb{R}): \int_{\mathbb{R}} v(x) u^{\prime}(x) \mathrm{d} x=0\right\} .
$$

We want to prove that the operator $\mathscr{L}$ is coercive on $\mathcal{X}$. This does not come for free, since $W^{\prime \prime}(u)$ is not bounded from below by any positive constant. However, we can prove the following

Lemma 5.3. Let $\mathscr{G}: H^{s}(\mathbb{R}) \rightarrow \mathbb{R}$ be defined by (5.5) and let $\mathcal{X} \subset H^{s}(\mathbb{R})$ be defined by (5.6). Then there exists a positive constant $C$ such that

$$
\mathscr{G}(v) \geqslant C\|v\|_{L^{2}(\mathbb{R})}^{2}, \quad \forall v \in \mathcal{X} .
$$


Proof. First of all, we observe that

$$
\mathscr{G}(v) \geqslant 0 \text { for any } v \in H^{s}(\mathbb{R}) .
$$

To prove it, for any $R>0$ we introduce the energy functional

$$
\mathscr{E}_{R}(w):=\frac{1}{2} \int_{Q_{R}} \frac{|w(x)-w(y)|^{2}}{|x-y|^{1+2 s}} \mathrm{~d} x \mathrm{~d} y+\int_{-R}^{R} W(w(x)) \mathrm{d} x,
$$

where $Q_{R}:=((-R, R) \times \mathbb{R}) \cup((\mathbb{R} \backslash(-R, R)) \times(-R, R))$. Since $u$ is a local minimum (see [19]), we have that $\mathscr{E}_{R}(u+\epsilon v) \geqslant \mathscr{E}_{R}(u)$ and $\mathscr{E}_{R}(u-\epsilon v) \geqslant \mathscr{E}_{R}(u)$ for any $\epsilon>0$ and any $v \in C_{0}^{\infty}([-R, R])$. As a consequence,

$$
\begin{aligned}
0 & \leqslant \mathscr{E}_{R}(u+\epsilon v)+\mathscr{E}_{R}(u-\epsilon v)-2 \mathscr{E}_{R}(u) \\
& =\epsilon^{2} D^{2} \mathscr{E}_{R}(u)[v, v]+o\left(\epsilon^{2}\right) \\
& =2 \epsilon^{2} \mathscr{G}(v)+o\left(\epsilon^{2}\right)
\end{aligned}
$$

Then, a division by $\epsilon^{2}$ and a limit argument gives that $\mathscr{G}(v) \geqslant 0$ for any $v \in$ $C_{0}^{\infty}([-R, R])$. Since $R>0$ is arbitrary this holds true for any $v \in C_{0}^{\infty}(\mathbb{R})$ and so, by density, for any $v \in H^{s}(\mathbb{R})$, thus proving (5.8).

Now we define $\mathcal{X}_{1}$ to be the set of all functions $v$ belonging to $\mathcal{X}$ and such that $\|v\|_{L^{2}(\mathbb{R})}=1$. Let also

$$
\bar{\imath}:=\inf _{v \in \mathcal{X}_{1}} \mathscr{G}(v)
$$

We remark that

$$
\bar{\imath}<+\infty \text {. }
$$

Indeed, let $I_{-}$and $I_{+}$be two disjoint open intervals and $\phi_{ \pm} \in C_{0}^{\infty}\left(\overline{I_{ \pm}}\right)$, with $\phi_{ \pm}>0$ in $I_{ \pm}$. We also define

$$
\begin{aligned}
& D:=\left(\int_{\mathbb{R}} \phi_{-}(x) u^{\prime}(x) \mathrm{d} x\right)^{2} \int_{\mathbb{R}} \phi_{+}^{2}(x) \mathrm{d} x+\left(\int_{\mathbb{R}} \phi_{+}(x) u^{\prime}(x) \mathrm{d} x\right)^{2} \int_{\mathbb{R}} \phi_{-}^{2}(x) \mathrm{d} x, \\
& a_{-}:=D^{-1 / 2} \int_{\mathbb{R}} \phi_{+}(x) u^{\prime}(x) \mathrm{d} x \\
& a_{+}:=D^{-1 / 2} \int_{\mathbb{R}} \phi_{-}(x) u^{\prime}(x) \mathrm{d} x,
\end{aligned}
$$

and $\bar{\phi}:=a_{-} \phi_{-}-a_{+} \phi_{+}$.

Notice that $\bar{\phi}$ is smooth and compactly supported, hence $\bar{\phi} \in H^{s}(\mathbb{R})$. Also, a simple computation shows that

$$
\begin{aligned}
& \int_{\mathbb{R}} \bar{\phi}(x) u^{\prime}(x) \mathrm{d} x=a_{-} \int_{\mathbb{R}} \phi_{-}(x) u^{\prime}(x) \mathrm{d} x-a_{+} \int_{\mathbb{R}} \phi_{+}(x) u^{\prime}(x) \mathrm{d} x=0 \text { and } \\
& \|\bar{\phi}\|_{L^{2}(\mathbb{R})}^{2}=a_{-}^{2} \int_{\mathbb{R}} \phi_{-}^{2}(x) \mathrm{d} x+a_{+}^{2} \int_{\mathbb{R}} \phi_{+}^{2}(x) \mathrm{d} x=1,
\end{aligned}
$$

and so $\bar{\phi} \in \mathcal{X}_{1}$. This implies that $\bar{\imath} \leqslant \mathscr{G}(\bar{\phi})$ that establishes (5.9).

From (5.8) we know that $\bar{\imath} \geqslant 0$. We claim that

$$
\bar{\imath}>0 \text {. }
$$


Before proving this, we observe that (5.7) would plainly follow from (5.10) since, for any $v \in \mathcal{X} \backslash\{0\}$,

$$
\mathscr{G}(v)=\|v\|_{L^{2}(\mathbb{R})}^{2} \mathscr{G}\left(v /\|v\|_{L^{2}(\mathbb{R})}\right) \geqslant \bar{\imath}\|v\|_{L^{2}(\mathbb{R})}^{2} .
$$

Hence, to prove the desired result it is enough to prove (5.10): for that we argue by contradiction ${ }^{4}$ and suppose that

$$
\bar{\imath}=0 .
$$

So, we take a minimizing sequence $\left\{v_{k}\right\}_{k} \subset \mathcal{X}_{1}$, that is, making use of (5.11),

$$
0=\bar{\imath}=\lim _{k \rightarrow+\infty} \mathscr{G}\left(v_{k}\right) .
$$

Of course, we can also assume, without loss of generality, that

$$
\mathscr{G}\left(v_{k}\right) \leqslant \bar{\imath}+1 \text {. }
$$

We claim that

$$
\left\|v_{k}\right\|_{H^{s}(\mathbb{R})} \text { is bounded uniformly in } k \text {. }
$$

To prove this, we use the assumptions on $W$ given in (1.2) and the asymptotic behavior of $u$ given by (5.1) to find a constant $\tilde{M}>0$ and a nonnegative smooth function $\varphi$ supported in a compact interval $\left[-K_{0}, K_{0}\right]$, with $K_{0}$ sufficiently large, such that

$$
W^{\prime \prime}(u)+\varphi \geqslant \tilde{M}>0, \quad \forall x \in \mathbb{R} .
$$

Thus, recalling (5.13), we obtain

$$
\begin{aligned}
\min \{1 / 2, \tilde{M} / 2\}\left\|v_{k}\right\|_{H^{s}(\mathbb{R})}^{2} \\
\leqslant \frac{1}{2} \int_{\mathbb{R}} \int_{\mathbb{R}} \frac{\left|v_{k}(x)-v_{k}(y)\right|^{2}}{|x-y|^{1+2 s}} \mathrm{~d} x \mathrm{~d} y+\frac{1}{2} \int_{\mathbb{R}}\left(W^{\prime \prime}(u)+\varphi\right) v_{k}^{2} \mathrm{~d} x \\
=\mathscr{G}\left(v_{k}\right)+\frac{1}{2} \int_{\mathbb{R}} \varphi v_{k}^{2} \mathrm{~d} x \\
\leqslant \bar{\imath}+1+\|\varphi\|_{L^{\infty}(\mathbb{R})} \int_{\mathbb{R}} v_{k}^{2} \mathrm{~d} x \\
=\bar{\imath}+1+\|\varphi\|_{L^{\infty}(\mathbb{R}) .}
\end{aligned}
$$

This establishes (5.14).

Now, we split the functional $\mathscr{G}$ as follows

$$
\mathscr{G}(v)=\frac{1}{2}\|v\|_{\mathcal{H}}^{2}+\mathscr{R}(v),
$$

where we set

$$
\begin{aligned}
\|v\|_{\mathcal{H}}^{2} & :=\int_{\mathbb{R}} \int_{\mathbb{R}} \frac{|v(x)-v(y)|^{2}}{|x-y|^{1+2 s}} \mathrm{~d} x \mathrm{~d} y+\int_{\mathbb{R}}\left(W^{\prime \prime}\left(u^{\prime}\right)+\varphi\right) v^{2} \mathrm{~d} x \\
\text { and } \quad \mathscr{R}_{(v)} & :=-\frac{1}{2} \int_{-K_{0}}^{K_{0}} \varphi v^{2} \mathrm{~d} x .
\end{aligned}
$$

\footnotetext{
${ }^{4}$ The proof is a bit long (it will end on page 19) and difficult to break into single pieces: for the facility of the reader, we provide the full details of all the arguments involved.
} 
In view of (5.15), the functional $\|\cdot\|_{\mathcal{H}}$ provides a norm which is equivalent to $\|\cdot\|_{H^{s}(\mathbb{R})}$ and which naturally arises from a scalar product (hence we call $\mathcal{H}$ the associated Hilbert space). Therefore, thanks to (5.14), there exists a function $v_{0} \in$ $H^{s}(\mathbb{R})$ such that, up to subsequences,

$$
v_{k} \rightarrow v_{0} \text { in } \mathcal{H},
$$

which gives

$$
\left\|v_{0}\right\|_{\mathcal{H}}^{2} \leqslant \liminf _{k \rightarrow \infty}\left\|v_{k}\right\|_{\mathcal{H}}^{2} .
$$

We remark that, at this level, we are not claiming that $v_{0} \in \mathcal{X}_{1}$, nor that $\mathscr{G}\left(v_{0}\right)=\bar{\imath}$ (i.e. we are not saying that $v_{0}$ is a minimizer).

From (5.14) and the compact embedding $H^{s}(\mathbb{R}) \hookrightarrow L^{2}([-K, K])$ for any compact subset $[-K, K] \subset \mathbb{R}$ (see, e. g., [8, Theorem 7.1]), we obtain that a strong convergence holds in (5.17) in the norm of $L^{2}([-K, K])$, namely

$$
v_{k} \rightarrow v_{0} \text { in } L^{2}([-K, K]) .
$$

This, by taking $K \geqslant K_{0}$, implies that

$$
\mathscr{R}\left(v_{k}\right) \rightarrow \mathscr{R}\left(v_{0}\right) .
$$

By (5.12) and (5.16) we know that

$$
0=\lim _{k \rightarrow+\infty} \mathscr{G}\left(v_{k}\right)=\lim _{k \rightarrow+\infty}\left(\frac{1}{2}\left\|v_{k}\right\|_{\mathcal{H}}^{2}+\mathscr{R}\left(v_{k}\right)\right) .
$$

On the other hand, by (5.15), we have that

$$
\left\|v_{k}\right\|_{\mathcal{H}}^{2} \geqslant \tilde{M}\left\|v_{k}\right\|_{L^{2}(\mathbb{R})}^{2}=\tilde{M}
$$

and so, recalling also (5.20), we deduce from (5.21) that

$$
0 \geqslant \tilde{M}+\mathscr{R}\left(v_{0}\right)
$$

In particular, since $\tilde{M}>0$ and $\mathscr{R}(0)=0$, this implies that

$$
v_{0} \not \equiv 0
$$

Now we claim that $v_{0} \in \mathcal{X}$, that is, by (5.6),

$$
\int_{\mathbb{R}} v_{0}(x) u^{\prime}(x) \mathrm{d} x=0
$$

To prove it, we notice that

$$
\int_{\mathbb{R}}\left(v_{0}(x)-v_{k}(x)\right)^{2} \mathrm{~d} x \leqslant 2 \int_{\mathbb{R}} v_{0}^{2}(x)+v_{k}^{2}(x) \mathrm{d} x \leqslant 2\left(\left\|v_{0}\right\|_{H^{s}(\mathbb{R})}^{2}+1\right) .
$$


Therefore, fixing $K>0$ (to be taken as large as we wish in the following) and using that $v_{k} \in \mathcal{X}$,

$$
\begin{aligned}
\mid \int_{\mathbb{R}} & v_{0}(x) u^{\prime}(x) \mathrm{d} x \mid \\
= & \left|\int_{\mathbb{R}}\left(v_{0}(x)-v_{k}(x)\right) u^{\prime}(x) \mathrm{d} x\right| \\
\leqslant & \left|\int_{-K}^{K}\left(v_{0}(x)-v_{k}(x)\right) u^{\prime}(x) \mathrm{d} x\right|+\left|\int_{\mathbb{R} \backslash[-K, K]}\left(v_{0}(x)-v_{k}(x)\right) u^{\prime}(x) \mathrm{d} x\right| \\
\leqslant & \sqrt{\int_{-K}^{K}\left(v_{0}(x)-v_{k}(x)\right)^{2} \mathrm{~d} x \int_{-K}^{K}\left(u^{\prime}(x)\right)^{2} \mathrm{~d} x} \\
& +\sqrt{\int_{\mathbb{R} \backslash[-K, K]}\left(v_{0}(x)-v_{k}(x)\right)^{2} \mathrm{~d} x \int_{\mathbb{R} \backslash[-K, K]}\left(u^{\prime}(x)\right)^{2} \mathrm{~d} x} \\
\leqslant & \left\|u^{\prime}\right\|_{L^{2}(\mathbb{R})}\left\|v_{0}-v_{k}\right\|_{L^{2}([-K, K])}+\left\|u^{\prime}\right\|_{L^{2}(\mathbb{R} \backslash[-K, K])} \sqrt{2\left(\left\|v_{0}\right\|_{H^{s}(\mathbb{R})}^{2}+1\right)} .
\end{aligned}
$$

So, if we fix $K>0$ and we send $k$ to $+\infty$, we deduce from (5.19) that

$$
\left|\int_{\mathbb{R}} v_{0}(x) u^{\prime}(x) \mathrm{d} x\right| \leqslant\left\|u^{\prime}\right\|_{L^{2}(\mathbb{R} \backslash[-K, K])} \sqrt{2\left(\left\|v_{0}\right\|_{H^{s}(\mathbb{R})}^{2}+1\right)} .
$$

Now we send $K$ to $+\infty$ and we finish the proof of (5.23).

Also, from (5.16), (5.18) and (5.20) we obtain

$$
\mathscr{G}\left(v_{0}\right) \leqslant \liminf _{k \rightarrow \infty} \mathscr{G}\left(v_{k}\right) .
$$

Hence, by (5.12), we obtain that $\mathscr{G}\left(v_{0}\right) \leqslant 0$ and so, by (5.8), that

$$
0=\mathscr{G}\left(v_{0}\right)=\min _{v \in H^{s}(\mathbb{R})} \mathscr{G}(v) .
$$

As a consequence $v_{0}$ solves the corresponding Euler-Lagrange equation $\mathscr{G}^{\prime}\left(v_{0}\right)=0$, that is

$$
L_{s} v_{0}=W^{\prime \prime}(u) v_{0} .
$$

The equation above should be, in principle, intended in the distributional sense: however, from the Morrey-Sobolev embedding (see, for instance, [8, Theorem 8.2]) we have that

$$
v_{0} \in C^{\alpha}(\mathbb{R}) \cap L^{\infty}(\mathbb{R})
$$

with $\alpha:=(2 s-1) / 2>0$ and so $v_{0}$ is also a classical solution of (5.25); see [20].

Now we point out that

$$
v_{0}(x) \rightarrow 0, \quad \text { as } x \rightarrow \pm \infty .
$$

Indeed, suppose by contradiction that there exist $\epsilon_{0}>0$ and a sequence of points $x_{n} \rightarrow+\infty$ such that $\left|v_{0}\left(x_{n}\right)\right|>\epsilon_{0}$. Then, by the uniform continuity in (5.26), we get that for every $n \in \mathbb{N}$ we can find a neighborhood $B_{n}$ of $x_{n}$ of measure $c_{0} \epsilon_{0}^{1 / \alpha}$, for a suitable $c_{0}>0$, such that $\left|v_{0}(x)\right|>\epsilon_{0} / 2$ for any $x \in B_{n}$. This contradicts the fact that $v_{0} \in L^{2}(\mathbb{R})$, thus proving (5.27). 
Now, from the assumptions on the potential $W$ in (1.2) and the asymptotic behavior of $u$ given in (5.1), we get that there exists $R>0$ such that

$$
W^{\prime \prime}(u(x)) \geqslant \tau:=W^{\prime \prime}(0) / 2>0 \quad \text { for every } x \in \mathbb{R} \backslash(-R, R) .
$$

Fixing $R$ in this way, using (5.26) and the fact that $u^{\prime}$ is positive (see (1.5)), we conclude that there exists a constant $\kappa_{0}>0$ such that $\left|v_{0}\right|<\kappa_{0} u^{\prime}$ in $[-R, R]$. Now, we denote by

$$
\kappa_{\star}:=\inf \left\{\kappa \text { s.t. }\left|v_{0}(x)\right|<\kappa u^{\prime}(x) \text { for any } x \in[-R, R]\right\} .
$$

Obviously, $\kappa_{\star} \leqslant \kappa_{0}$. Then,

$$
\left|v_{0}\right| \leqslant \kappa_{\star} u^{\prime} \quad \text { in }[-R, R]
$$

and

$$
\text { there exists } x^{\star} \in[-R, R] \text { such that }\left|v_{0}\left(x^{\star}\right)\right|=\kappa_{\star} u^{\prime}\left(x^{\star}\right) .
$$

We define the functions

$$
z_{ \pm}(x):=\kappa_{\star} u^{\prime}(x) \pm v_{0}(x), \quad \forall x \in \mathbb{R} .
$$

Notice that, by (5.29), we have

$$
z_{ \pm} \geqslant 0 \text { in }[-R, R]
$$

Moreover, from (1.5) we have that $u^{\prime}$ solves

$$
L_{s} u^{\prime}=W^{\prime \prime}(u) u^{\prime},
$$

which, together with (5.25) implies that $z_{ \pm}$satisfy

$$
L_{s} z_{ \pm}=W^{\prime \prime}(u) z_{ \pm} \quad \text { in } \mathbb{R} \text {. }
$$

We claim that we can extend the validity of (5.31) to the whole of $\mathbb{R}$, namely

$$
z_{ \pm} \geqslant 0 \text { in } \mathbb{R} \text {. }
$$

To prove (5.33), we assume by contradiction that $\inf _{\mathbb{R}} z_{ \pm}<0$. We notice that $z_{ \pm}$ are continuous functions and that, thanks to (1.5) and (5.27),

$$
z_{ \pm}(x) \rightarrow 0, \quad \text { as } x \rightarrow \pm \infty \text {. }
$$

Therefore, since (5.31) holds, there exist $x_{ \pm} \in \mathbb{R} \backslash[-R, R]$ such that

$$
z_{ \pm}\left(x_{ \pm}\right)=\min _{\mathbb{R}} z_{ \pm}<0 .
$$

Hence

$$
L_{s} z_{ \pm}\left(x_{ \pm}\right)=\frac{1}{2} \int_{\mathbb{R}} \frac{z_{ \pm}\left(x_{ \pm}+y\right)+z_{ \pm}\left(x_{ \pm}-y\right)-2 z_{ \pm}\left(x_{ \pm}\right)}{|y|^{1+2 s}} \mathrm{~d} y \geqslant 0,
$$

since $z_{ \pm}(x) \geqslant z_{ \pm}\left(x_{ \pm}\right)$for any $x \in \mathbb{R}$. On the other hand, since $x_{ \pm} \in \mathbb{R} \backslash[-R, R]$, we deduce from (5.28) that $W^{\prime \prime}\left(u\left(x_{ \pm}\right)\right)>0$, and so, by (5.32) and (5.34), we have

$$
L_{s} z_{ \pm}\left(x_{ \pm}\right)=W^{\prime \prime}\left(u\left(x_{ \pm}\right)\right) z_{ \pm}\left(x_{ \pm}\right)<0
$$

thus obtaining a contradiction with (5.35). This proves (5.33).

Now we define, for every $x \in \mathbb{R}$,

$$
d_{1}(x):=\max \left\{W^{\prime \prime}(u(x)), \tau\right\}, \quad d_{2}(x):=d_{1}(x)-W^{\prime \prime}(u(x)),
$$


where $\tau$ was introduced in (5.28). Notice that both $d_{1}$ and $d_{2}$ are nonnegative on $\mathbb{R}$. Therefore, recalling (5.32) and (5.33), we have that $z_{ \pm}$satisfy

$$
L_{s} z_{ \pm}=W^{\prime \prime}(u) z_{ \pm}=\left(W^{\prime \prime}(u)-d_{1}\right) z_{ \pm}+d_{1} z_{ \pm}=-d_{2} z_{ \pm}+d_{1} z_{ \pm} \leqslant d_{1} z_{ \pm} .
$$

From (5.33), (5.36) and Proposition 4.1 we obtain that

$$
\text { either } z_{ \pm}>0 \text { or } z_{ \pm} \equiv 0
$$

Then, from (5.30) and (5.37), we deduce that either $z_{-} \equiv 0$ or $z_{+} \equiv 0$. In any case, there exists $\kappa_{\sharp} \in\left\{\kappa_{\star},-\kappa_{\star}\right\}$ such that $\kappa_{\sharp} u^{\prime}(x)=v_{0}(x)$ for any $x \in \mathbb{R}$. Hence, from (5.23), we have that $\kappa_{\sharp}$ has to be zero, and so $v_{0} \equiv 0$, which is a contradiction with (5.22).

Hence, (5.10) holds true, and this concludes the proof of Lemma 5.3.

A simple consequence of Lemma 5.3 is the following result, in which the norm in $L^{2}(\mathbb{R})$ is replaced by the one in $H^{s}(\mathbb{R})$ :

Lemma 5.4. Let $\mathscr{G}: H^{s}(\mathbb{R}) \rightarrow \mathbb{R}$ be defined by $(5.5)$ and let $\mathcal{X} \subset H^{s}(\mathbb{R})$ be defined by (5.6). Then there exists a positive constant $C$ such that

$$
\mathscr{G}(v) \geqslant C\|v\|_{H^{s}(\mathbb{R})}^{2}, \quad \forall v \in \mathcal{X} .
$$

Proof. Let $C$ be the constant given by Lemma 5.3. Thanks to the boundedness of $W^{\prime \prime}$, there exists $\delta>0$ small enough such that

$$
2(1-\delta) C+\delta W^{\prime \prime}(u)>C \text {. }
$$

This, together with (5.7), yields

$$
\begin{aligned}
\mathscr{G}(v) & =(1-\delta) \mathscr{G}(v)+\delta \mathscr{G}(v) \\
& \geqslant(1-\delta) C\|v\|_{L^{2}(\mathbb{R})}^{2}+\delta \mathscr{G}(v) \\
& =\frac{\delta}{2} \int_{\mathbb{R}} \int_{\mathbb{R}} \frac{|v(x)-v(y)|^{2}}{|x-y|^{1+2 s}} \mathrm{~d} x \mathrm{~d} y+\frac{1}{2} \int_{\mathbb{R}}\left(2(1-\delta) C+\delta W^{\prime \prime}(u)\right) v^{2} \mathrm{~d} x \\
& \geqslant \min \{\delta / 2, C / 2\}\|v\|_{H^{s}(\mathbb{R})}^{2},
\end{aligned}
$$

and thus in turn implying the desired result, up to renaming $C$.

Proof of Theorem 5.2. In order to find a solution to (5.2), we set

$$
f:=u^{\prime}+\eta\left(W^{\prime \prime}(u)-W^{\prime \prime}(0)\right)
$$


where $\eta$ is given in (5.3). We observe that $f$ belongs to $\mathcal{X}$, which is given in (5.6). Indeed, thanks to (1.2) and (5.1), $f \in H^{s}(\mathbb{R})$. Moreover,

$$
\begin{aligned}
\int_{\mathbb{R}} f(x) & u^{\prime}(x) \mathrm{d} x \\
= & \int_{\mathbb{R}}\left(u^{\prime}(x)\right)^{2} \mathrm{~d} x+\eta \int_{\mathbb{R}} W^{\prime \prime}(u(x)) u^{\prime}(x) \mathrm{d} x-\eta W^{\prime \prime}(0) \int_{\mathbb{R}} u^{\prime}(x) \mathrm{d} x \\
= & \int_{\mathbb{R}}\left(u^{\prime}(x)\right)^{2} \mathrm{~d} x+\eta \int_{\mathbb{R}} \frac{\mathrm{d}}{\mathrm{d} x} W^{\prime}(u(x)) \mathrm{d} x-\eta W^{\prime \prime}(0) \\
& =\int_{\mathbb{R}}\left(u^{\prime}(x)\right)^{2} \mathrm{~d} x+\eta\left(W^{\prime}(1)-W^{\prime}(0)\right)-\frac{\int_{\mathbb{R}}\left(u^{\prime}(x)\right)^{2} \mathrm{~d} x}{W^{\prime \prime}(0)} W^{\prime \prime}(0) \\
& =0 .
\end{aligned}
$$

Now, we consider the continuous linear functional $F$ on $H^{s}(\mathbb{R})$ given by

$$
v \longmapsto F v:=-\int_{\mathbb{R}} f v \mathrm{~d} x \quad \forall v \in H^{s}(\mathbb{R}) .
$$

The bilinear form $\mathscr{L}$ defined in (5.4) is coercive in $\mathcal{X}$, thanks to Lemma 5.4. Therefore, from Lax-Milgram Theorem, there exists a unique $\psi \in H^{s}(\mathbb{R})$ such that

$$
\mathscr{L}(\psi, w)=F w \text { for any } w \in H^{s}(\mathbb{R}) .
$$

This means that $\psi$ is a weak solution to (5.2). As a matter of fact, by the MorreySobolev embedding (see, for instance, [8, Theorem 8.2]), we have that $\psi$ is bounded and continuous and so it is also a classical solution of (5.2), see [20]. Since $s>1 / 2$, the fractional Morrey embedding (see, e.g., Theorem 8.2 in [8]) implies that it also belongs to the Hölder space $C^{(2 s-1) / 2}(\mathbb{R})$. This and the fact that $\psi \in L^{2}(\mathbb{R})$ give that $\psi$ tends to zero at $\pm \infty$.

Finally, from the regularity results in [21, 22] (see, also, [3, Theorem 5] and [19, Appendix 6.1]), we have that $\psi \in C_{\text {loc }}^{1, \alpha}(\mathbb{R})$, for some $\alpha=\alpha(s) \in(0,1)$ and $\psi^{\prime} \in$ $L^{\infty}(\mathbb{R})$. This concludes the proof of Theorem 5.2.

\section{Auxiliary layer solutions}

In order to improve the asymptotics for the layer solution $u$ given in (5.1), we consider an even and positive function $\omega \in C^{\infty}(\mathbb{R})$ such that

$$
\omega(x)=\frac{1}{|x|^{1+2 s}} \quad \text { for any }|x|>1,
$$

and we set

$$
A:=\int_{0}^{+\infty} \omega(t) \mathrm{d} t
$$

Then we define a function $\phi \in C^{\infty}(\mathbb{R})$ given by

$$
\phi(x)=\frac{1}{2}\left(1+\frac{1}{A} \int_{0}^{x} \omega(t) \mathrm{d} t\right) .
$$

We notice that

$$
\phi^{\prime}(x)=\frac{1}{2 A} \omega(x)
$$


hence $\phi$ is strictly increasing and

$$
\phi^{\prime}(x)=\frac{1}{2 A} \frac{1}{|x|^{1+2 s}} \quad \text { for any }|x|>1 .
$$

Moreover, $\phi$ is such that $\phi(-\infty)=0, \phi(0)=1 / 2$ and $\phi(+\infty)=1$ and, since $\omega$ is even, we have the following symmetry property for $\phi$ :

$$
\begin{aligned}
\phi(-x) & =\frac{1}{2}+\frac{1}{2 A} \int_{0}^{-x} \omega(t) \mathrm{d} t \\
& =1-\frac{1}{2}-\frac{1}{2 A} \int_{0}^{x} \omega(\tau) \mathrm{d} \tau \\
& =1-\phi(x) .
\end{aligned}
$$

We notice that, thanks to (6.2),

$$
\frac{L_{s} \phi^{\prime}(x)}{\phi^{\prime}(x)}=\frac{L_{s} \frac{1}{2 A} \omega(x)}{\frac{1}{2 A} \omega(x)}=\frac{L_{s} \omega(x)}{\omega(x)} .
$$

We define the functions

$$
\begin{aligned}
& g(t):=L_{s} \phi(t), \quad t \in \mathbb{R}, \\
& h(r):=g\left(\phi^{-1}(r)\right), \quad r \in(0,1),
\end{aligned}
$$

and the potential

$$
V(r):=\int_{0}^{r} h(\rho) \mathrm{d} \rho, \quad r \in(0,1) .
$$

In this way, the function $\phi$ satisfies the equation

$$
L_{s} \phi-V^{\prime}(\phi)=0 \quad \text { in } \mathbb{R} .
$$

The following lemma explicitly computes the asymptotic behavior of $L_{s} \omega$ and will play a crucial role in the proof of the forthcoming Proposition 6.2:

Lemma 6.1. Let $\omega$ be as introduced in the beginning of Section 6. Then

$$
\lim _{|x| \rightarrow+\infty} \frac{L_{s} \omega(x)}{\omega(x)}=\int_{\mathbb{R}} \omega(z) \mathrm{d} z .
$$

Proof. For any $x, z \in \mathbb{R}$ with $|x| \geqslant 2$, we define the quantity

$$
i(x, z):=\frac{\omega(z)-\omega(x)-\chi_{(-1 / 4,1 / 4)}(x-z) \omega^{\prime}(x)(z-x)}{|x-z|^{1+2 s}} .
$$

We have that, for any $z \in \mathbb{R}$,

$$
\lim _{|x| \rightarrow+\infty}|x|^{1+2 s} i(x, z)=\lim _{|x| \rightarrow+\infty} \frac{|x|^{1+2 s}}{|x-z|^{1+2 s}}(\omega(z)-\omega(x))=\omega(z) .
$$

Also, if $|z| \leqslant 1$ and $|x| \geqslant 2$, we have that $|x-z| \geqslant|x|-|z| \geqslant|x| / 2$, and therefore

$$
|x|^{1+2 s}|i(x, z)|=\frac{|x|^{1+2 s}|\omega(x)-\omega(z)|}{|x-z|^{1+2 s}} \leqslant 16 \sup _{\mathbb{R}}|\omega| .
$$

From (6.8), (6.9) and the Bounded Convergence Theorem, we conclude that

$$
\lim _{|x| \rightarrow+\infty}|x|^{1+2 s} \int_{-1}^{1} i(x, z) \mathrm{d} z=\int_{-1}^{1} \lim _{|x| \rightarrow+\infty}|x|^{1+2 s} i(x, z) \mathrm{d} z=\int_{-1}^{1} \omega(z) \mathrm{d} z .
$$


Now, fixed $|x| \geqslant 2$, we estimate the contribution in $\mathbb{R} \backslash(-1,1)$. For this, we write $\mathbb{R} \backslash(-1,1)=P \cup Q \cup R \cup S$, where

$$
\begin{aligned}
& P=\{z \in \mathbb{R} \backslash(-1,1):|x| / 2<|z| \leqslant 2|x| \text { and }|x-z| \geqslant 1 / 4\}, \\
& Q=\{z \in \mathbb{R} \backslash(-1,1):|x| / 2<|z| \leqslant 2|x| \text { and }|x-z|<1 / 4\}, \\
& R=\{z \in \mathbb{R} \backslash(-1,1):|z|>2|x|\}, \\
& S=\{z \in \mathbb{R} \backslash(-1,1):|z| \leqslant|x| / 2\} .
\end{aligned}
$$

Let us estimate the contribution coming from $P$ : if $z \in P$, then

$$
\begin{aligned}
|i(x, z)| & =\frac{|\omega(z)-\omega(x)|}{|x-z|^{1+2 s}} \leqslant \frac{|\omega(z)|+|\omega(x)|}{|x-z|^{1+2 s}}=\frac{\left(1 /|z|^{1+2 s}\right)+\left(1 /|x|^{1+2 s}\right)}{|x-z|^{1+2 s}} \\
& \leqslant \frac{2^{1+2 s}+1}{|x|^{1+2 s}|x-z|^{1+2 s}} .
\end{aligned}
$$

On the other hand, since $\omega$ is even in $\mathbb{R} \backslash(-1,1)$, we have

$$
\omega(z)-\omega(x)=\omega(|z|)-\omega(|x|)=\omega^{\prime}(\xi)(|z|-|x|),
$$

where $\xi=t|z|+(1-t)|x| \geqslant t \frac{|x|}{2}+(1-t) \frac{|x|}{2}=\frac{|x|}{2}$, for $t \in(0,1)$. Therefore,

$$
|\omega(z)-\omega(x)| \leqslant\left|\omega^{\prime}(\xi)\right||| z|-| x|| \leqslant \frac{C}{|x|^{2+2 s}}|z-x| .
$$

This implies that

$$
|i(x, z)|=\frac{|\omega(z)-\omega(x)|}{|x-z|^{1+2 s}} \leqslant \frac{C}{|x|^{2+2 s}|x-z|^{2 s}} .
$$

Now we make a simple interpolation argument. Namely, we observe that for any $X, Y \geqslant 0$ and any $\alpha, \beta \in(0,1)$ such that $\alpha+\beta=1$ we have

$$
\min \{X, Y\} \leqslant X^{\alpha} Y^{\beta} \text {. }
$$

Therefore

(6.13) $\min \left\{X^{2+2 s} Y^{2 s}, X^{1+2 s} Y^{1+2 s}\right\}=X^{1+2 s} Y^{2 s} \min \{X, Y\} \leqslant X^{1+2 s+\alpha} Y^{2 s+\beta}$.

So, putting together (6.11) and (6.12) and using (6.13) with $X:=1 /|x|$ and $Y:=$ $1 /|x-z|$, we have that, for every $z \in P$ and $\alpha, \beta \in(0,1)$ such that $\alpha+\beta=1$,

$$
|i(x, z)| \leqslant \frac{C}{|x|^{1+2 s+\alpha}|x-z|^{\beta+2 s}} .
$$

Therefore,

$$
\begin{aligned}
|x|^{1+2 s} \int_{P}|i(x, z)| \mathrm{d} z & \leqslant \frac{C}{|x|^{\alpha}} \int_{P} \frac{\mathrm{d} z}{|x-z|^{\beta+2 s}} \\
& \leqslant \frac{C}{|x|^{\alpha}} \int_{\{|x-z| \geqslant 1 / 4\}} \frac{\mathrm{d} z}{|x-z|^{\beta+2 s}} .
\end{aligned}
$$

In particular, we choose $\alpha=s$ and $\beta=1-s$ and we get

$$
|x|^{1+2 s} \int_{P}|i(x, z)| \mathrm{d} z \leqslant \frac{C}{|x|^{s}} \int_{\{|x-z| \geqslant 1 / 4\}} \frac{\mathrm{d} z}{|x-z|^{1+s}} \leqslant \frac{C}{|x|^{s}} .
$$


Now, if $z \in Q$, we use the Taylor expansion of the function $1 /|x|^{1+2 s}$ to get

$$
\begin{aligned}
\omega(z)-\omega(x)-\chi_{(-1 / 4,1 / 4)} & (x-z) \omega^{\prime}(x)(z-x) \\
= & \omega(z)-\omega(x)-\omega^{\prime}(x)(z-x) \\
& =\frac{1}{|z|^{1+2 s}}-\frac{1}{|x|^{1+2 s}}+\frac{1+2 s}{|x|^{3+2 s}} x(z-x) \\
& =\frac{(1+2 s)(2+2 s)}{|\xi|^{3+2 s}}|x-z|^{2},
\end{aligned}
$$

where $\xi$ is a suitable point lying on the segment joining $x$ to $z$. We notice that both $x$ and $z$ lie either in $[|x| / 2,+\infty)$ or in $(-\infty,-|x| / 2]$, because $z \geqslant 0$ if and only if $x \geqslant 0$, if $z \in Q$. This implies that $|\xi| \geqslant|x| / 2$, and therefore

$$
\begin{aligned}
|i(x, z)| & =\frac{\left|\omega(z)-\omega(x)-\chi_{(-1 / 4,1 / 4)}(x-z) \omega^{\prime}(x)(z-x)\right|}{|x-z|^{1+2 s}} \\
& =\frac{C}{|\xi|^{3+2 s}}|x-z|^{1-2 s} \leqslant \frac{C}{|x|^{3+2 s}}|x-z|^{1-2 s} .
\end{aligned}
$$

Hence,

$$
\begin{aligned}
|x|^{1+2 s} \int_{Q}|i(x, z)| \mathrm{d} z & \leqslant \frac{C}{|x|^{2}} \int_{Q}|x-z|^{1-2 s} \mathrm{~d} z \\
& \leqslant \frac{C}{|x|^{2}} \int_{\{|x-z|<1 / 4\}}|x-z|^{1-2 s} \mathrm{~d} z \leqslant \frac{C}{|x|^{2}} .
\end{aligned}
$$

Moreover, if $z \in R$, we have that $|x-z| \geqslant|z|-|x| \geqslant|x|>1 / 4$, so we can estimate $|i(x, z)|$ as in (6.11). Therefore,

$$
\begin{aligned}
|x|^{1+2 s} \int_{R}|i(x, z)| \mathrm{d} z & \leqslant C \int_{\{|z| \geqslant 2|x|\}} \frac{\mathrm{d} z}{|x-z|^{1+2 s}} \\
& \leqslant C \int_{\{|x-z| \geqslant|x|\}} \frac{\mathrm{d} z}{|x-z|^{1+2 s}}=\frac{C}{|x|^{2 s}} .
\end{aligned}
$$

Finally, we compute the contribution coming from $S$ for large $|x|$ :

$$
\begin{aligned}
\frac{\int_{S} i(x, z) \mathrm{d} z}{\omega(x)} & =|x|^{1+2 s} \int_{\{1 \leqslant|z| \leqslant|x| / 2\}} \frac{\omega(z)-\omega(x)}{|x-z|^{1+2 s}} \mathrm{~d} z \\
& =|x|^{1+2 s} \int_{\{1 \leqslant|z| \leqslant|x| / 2\}} \frac{1 /|z|^{1+2 s}-1 /|x|^{1+2 s}}{|x-z|^{1+2 s}} \mathrm{~d} z \\
& =\int_{\{1 \leqslant|z| \leqslant|x| / 2\}} \frac{|x|^{1+2 s}-|z|^{1+2 s}}{|z|^{1+2 s}|x-z|^{1+2 s}} \mathrm{~d} z .
\end{aligned}
$$

Now, setting $t=z / x$, we have that

$$
\frac{|x|^{1+2 s}-|z|^{1+2 s}}{|x-z|^{1+2 s}}=\frac{|x|^{1+2 s}\left(1-\left|\frac{z}{x}\right|^{1+2 s}\right)}{|x|^{1+2 s}\left|1-\frac{z}{x}\right|^{1+2 s}}=\frac{1-|t|^{1+2 s}}{|1-t|^{1+2 s}},
$$

which is bounded for $|t| \leqslant 1 / 2$. Therefore, we notice that the quantity

$$
\frac{|x|^{1+2 s}-|z|^{1+2 s}}{|x-z|^{1+2 s}}
$$


is uniformly bounded in $x$ in the set $S$ and tends to 1 as $|x| \rightarrow+\infty$, and $\frac{1}{|z|^{1+2 s}} \in$ $L^{1}(\mathbb{R} \backslash(-1,1))$. Therefore, by the Dominated Convergence Theorem, we have that

$$
\lim _{|x| \rightarrow+\infty} \frac{\int_{S} i(x, z) \mathrm{d} z}{\omega(x)}=\int_{\{|z| \geqslant 1\}} \frac{\mathrm{d} z}{|z|^{1+2 s}}=\int_{\{|z| \geqslant 1\}} \omega(z) \mathrm{d} z .
$$

Putting together (6.10), (6.14), (6.15), (6.16) and (6.17), we obtain that

$$
\lim _{|x| \rightarrow+\infty} \frac{L_{s} \omega(x)}{\omega(x)}=\int_{-1}^{1} \omega(z) \mathrm{d} z+\int_{|z| \geqslant 1} \omega(z) \mathrm{d} z=\int_{\mathbb{R}} \omega(z) \mathrm{d} z .
$$

Now, we can prove the following ${ }^{5}$ :

Proposition 6.2. The potential $V$ satisfies the following properties:

i) $V^{\prime}(0):=\lim _{r \rightarrow 0^{+}} V^{\prime}(r)=0$,

ii) $V^{\prime \prime}(0):=\lim _{r \rightarrow 0^{+}} V^{\prime \prime}(r)=2 A$, where $A$ is defined in (6.1).

Proof. First we point out that, by the definitions in (6.6) and (6.7), we have that $V^{\prime}(r)=h(r)$ and

$$
V^{\prime \prime}(r)=h^{\prime}(r)=\frac{g^{\prime}\left(\phi^{-1}(r)\right)}{\phi^{\prime}\left(\phi^{-1}(r)\right)}=\frac{L_{s} \phi^{\prime}\left(\phi^{-1}(r)\right)}{\phi^{\prime}\left(\phi^{-1}(r)\right)},
$$

Let us show $i$ ). We have

$$
\lim _{r \rightarrow 0^{+}} V^{\prime}(r)=\lim _{r \rightarrow 0^{+}} h(r)=\lim _{x \rightarrow-\infty} L_{s} \phi(x) .
$$

So we have to prove that the limit in (6.19) exists and is equal to zero. For this, we fix $R>1$ and we compute

$$
\begin{aligned}
& \left|\int_{\mathbb{R}} \frac{\phi(x+y)+\phi(x-y)-2 \phi(x)}{|y|^{1+2 s}} \mathrm{~d} y\right| \\
= & \left|\int_{B_{R}} \frac{\phi(x+y)+\phi(x-y)-2 \phi(x)}{|y|^{1+2 s}} \mathrm{~d} y+\int_{\mathbb{R} \backslash B_{R}} \frac{\phi(x+y)+\phi(x-y)-2 \phi(x)}{|y|^{1+2 s}} \mathrm{~d} y\right| \\
\leqslant & C\left(\int_{B_{R}} \frac{\sup _{B_{R}(x)}\left|\phi^{\prime \prime}\right|}{|y|^{2 s-1}} \mathrm{~d} y+\int_{\mathbb{R} \backslash B_{R}} \frac{\|\phi\|_{L^{\infty}(\mathbb{R})}}{|y|^{1+2 s}} \mathrm{~d} y\right) \\
= & C\left(R^{2-2 s} \sup _{B_{R}(x)}\left|\phi^{\prime \prime}\right|+R^{-2 s}\|\phi\|_{L^{\infty}(\mathbb{R})}\right) .
\end{aligned}
$$

Now, fixed $R$, we send $x \rightarrow-\infty$, obtaining that the first term tends to zero, thanks to (6.3). Then, sending $R \rightarrow+\infty$, we have that also the second term tends to zero. This concludes the proof of $i$ ).

Now, we show $i i)$. Recalling (6.18) and (6.5), we have that

$$
\lim _{r \rightarrow 0^{+}} V^{\prime \prime}(r)=\lim _{x \rightarrow-\infty} \frac{L_{s} \phi^{\prime}(x)}{\phi^{\prime}(x)}=\lim _{x \rightarrow-\infty} \frac{L_{s} \omega(x)}{\omega(x)} .
$$

Hence, the desired result follows from Lemma 6.1 and (6.1).

\footnotetext{
${ }^{5}$ We remark that $V$ is $C^{\infty}$ since so is $\phi$ (recall (6.6) and (6.7)). As usual, in the statement of Proposition 6.2 , we are using the abuse of notation to identify $V^{\prime}(0)$ and $V^{\prime \prime}(0)$ with their limit values, once we prove that these limits exist. Of course, by symmetry, a similar computation could have done for $V^{\prime}(1)$ and $V^{\prime \prime}(1)$.
} 
Moreover, the following holds:

Proposition 6.3. The potential $V$ is symmetric with respect to $1 / 2$.

Proof. We compute, for any $r \in(0,1 / 2)$,

$$
\begin{aligned}
V\left(\frac{1}{2}+r\right) & -V\left(\frac{1}{2}-r\right) \\
& =\int_{0}^{1 / 2+r} h(\rho) \mathrm{d} \rho-\int_{0}^{1 / 2-r} h(\rho) \mathrm{d} \rho \\
& =\int_{0}^{1 / 2} h(\rho) \mathrm{d} \rho+\int_{1 / 2}^{1 / 2+r} h(\rho) \mathrm{d} \rho-\int_{0}^{1 / 2} h(\rho) \mathrm{d} \rho+\int_{1 / 2-r}^{1 / 2} h(\rho) \mathrm{d} \rho \\
& =\int_{1 / 2-r}^{1 / 2+r} h(\rho) \mathrm{d} \rho .
\end{aligned}
$$

Hence, we have to show that the function $h$ is odd with respect to $1 / 2$, that is

$$
h\left(\frac{1}{2}+r\right)+h\left(\frac{1}{2}-r\right)=0 .
$$

To prove $(6.21)$, we set $\theta(r):=\phi^{-1}(1 / 2+r)$. Notice that, by applying (6.4) with $x:=\theta(r)$ we have that

$$
\phi(-\theta(r))=1-\phi(\theta(r))=1-\left(\frac{1}{2}+r\right)=\frac{1}{2}-r
$$

and so

$$
\phi^{-1}\left(\frac{1}{2}-r\right)=-\theta(r)
$$


Hence we can compute

$$
\begin{aligned}
h\left(\frac{1}{2}+r\right)+ & h\left(\frac{1}{2}-r\right) \\
= & g\left(\phi^{-1}\left(\frac{1}{2}+r\right)\right)+g\left(\phi^{-1}\left(\frac{1}{2}-r\right)\right) \\
= & g(\theta(r))+g(-\theta(r)) \\
= & L_{s} \phi(\theta(r))+L_{s} \phi(-\theta(r)) \\
= & \frac{1}{2} \int_{\mathbb{R}} \frac{\phi(\theta(r)+y)+\phi(\theta(r)-y)-2 \phi(\theta(r))}{|y|^{1+2 s}} \mathrm{~d} y \\
& +\frac{1}{2} \int_{\mathbb{R}} \frac{\phi(-\theta(r)+y)+\phi(-\theta(r)-y)-2 \phi(-\theta(r))}{|y|^{1+2 s}} \mathrm{~d} y \\
= & \frac{1}{2} \int_{\mathbb{R}} \frac{\phi(\theta(r)+y)+\phi(\theta(r)-y)-2 \phi(\theta(r))}{|y|^{1+2 s}} \mathrm{~d} y \\
& +\frac{1}{2} \int_{\mathbb{R}} \frac{1-\phi(\theta(r)-y)+1-\phi(\theta(r)+y)-2[1-\phi(\theta(r))]}{|y|^{1+2 s}} \mathrm{~d} y \\
= & \frac{1}{2} \int_{\mathbb{R}} \frac{\phi(\theta(r)+y)+\phi(\theta(r)-y)-2 \phi(\theta(r))}{|y|^{1+2 s}} \mathrm{~d} y \\
& -\frac{1}{2} \int_{\mathbb{R}} \frac{\phi(\theta(r)+y)+\phi(\theta(r)-y)-2 \phi(\theta(r))}{|y|^{1+2 s}} \mathrm{~d} y \\
= & 0,
\end{aligned}
$$

where we have used (6.4) once again. This proves (6.21) and concludes the proof of Proposition 6.3.

Now, for any $a>0$, we set $\mu_{a}=\left(V^{\prime \prime}(0) a\right)^{-1 / 2 s}$ and we define the function

$$
\phi_{a}(x):=\phi\left(\mu_{a} x\right)
$$

and the potential $V_{a}=\mu_{a}^{2 s} V$. Then, the function $\phi_{a}$ satisfies

$$
L_{s} \phi_{a}(x)=\mu_{a}^{2 s} L_{s} \phi\left(\mu_{a} x\right)=\mu_{a}^{2 s} V^{\prime}\left(\phi\left(\mu_{a} x\right)\right)=\mu_{a}^{2 s} V^{\prime}\left(\phi_{a}(x)\right)=V_{a}^{\prime}\left(\phi_{a}(x)\right) .
$$

Moreover,

$$
V_{a}^{\prime \prime}(0)=\mu_{a}^{2 s} V^{\prime \prime}(0)=\frac{1}{a}
$$

Since $W^{\prime \prime}(0)>0$ (see the assumptions in (1.2)), we can say that there exists $a_{0}>0$ such that

$$
V_{a_{0}}^{\prime \prime}(0)=W^{\prime \prime}(0), \text { namely } a_{0}:=\frac{1}{W^{\prime \prime}(0)} .
$$

Now, we prove the following

Proposition 6.4. There exists a positive constant $C$, depending on $s, a_{0}$ and $A$, such that the following estimates hold:

$$
\left|\phi_{a_{0}}(x)-H(x)\right| \leqslant \frac{C}{1+|x|^{2 s}} \quad \text { for any } x \in \mathbb{R}
$$


and

$$
\left|\phi_{a_{0}}(x)-H(x)+\frac{a_{0}}{2 s} \frac{x}{|x|^{1+2 s}}\right| \leqslant \frac{C}{|x|^{1+2 s}} \quad \text { for any } x \in \mathbb{R} \text {. }
$$

Proof. First, we show that, for any $|x|>1$,

$$
\phi(x)-H(x)+\frac{1}{4 s A} \frac{x}{|x|^{1+2 s}}=0 .
$$

For this, we consider the case $x>1$, since the case $x<-1$ is similar, and we compute

$$
\begin{aligned}
\phi(x)-H(x)+\frac{1}{4 s A} \frac{x}{|x|^{1+2 s}} & =\phi(x)-1+\frac{1}{4 s A} \frac{x}{|x|^{1+2 s}} \\
& =-\int_{x}^{+\infty} \phi^{\prime}(t) d t+\frac{1}{4 s A} \frac{x}{|x|^{1+2 s}} \\
& =-\frac{1}{2 A} \int_{x}^{+\infty} \frac{1}{|t|^{1+2 s}} d t+\frac{1}{4 s A} \frac{x}{|x|^{1+2 s}} \\
& =-\frac{1}{4 s A} \frac{x}{|x|^{1+2 s}}+\frac{1}{4 s A} \frac{x}{|x|^{1+2 s}}=0 .
\end{aligned}
$$

This proves (6.28).

In turn, (6.28) implies that, for $|x|>1$,

$$
|\phi(x)-H(x)|=\frac{1}{4 s A} \frac{1}{|x|^{2 s}} \leqslant \frac{C}{1+|x|^{2 s}} .
$$

Moreover, for $|x|<1$ we have

$$
|\phi(x)-H(x)| \leqslant 2 \leqslant \frac{4}{1+|x|^{2 s}} .
$$

Putting together the last two inequalities and taking $x=\mu_{a_{0}} y$ (recall (6.22)), we obtain (6.26).

To show (6.27), we observe that, if $|x| \leqslant 1$,

$$
\begin{aligned}
\left|\phi(x)-H(x)+\frac{1}{4 s A} \frac{x}{|x|^{1+2 s}}\right| & \leqslant 2+\frac{1}{4 s A} \frac{1}{|x|^{2 s}} \leqslant\left(2+\frac{1}{4 s A}\right) \frac{1}{|x|^{2 s}} \\
& \leqslant\left(2+\frac{1}{4 s A}\right) \frac{1}{|x|^{1+2 s}} .
\end{aligned}
$$

Putting together (6.28) and (6.29), we obtain

$$
\left|\phi(x)-H(x)+\frac{1}{4 s A} \frac{x}{|x|^{1+2 s}}\right| \leqslant\left(2+\frac{1}{4 s A}\right) \frac{1}{|x|^{1+2 s}} \quad \text { for any } x \in \mathbb{R} \text {. }
$$

Now, we take $x=\mu_{a_{0}} y$ in (6.30) and, recalling (6.22), we get

$$
\left|\phi_{a_{0}}(y)-H(y)+\frac{1}{4 s A \mu_{a_{0}}^{2 s}} \frac{y}{|y|^{1+2 s}}\right| \leqslant\left(2+\frac{1}{4 s A}\right) \frac{1}{\mu_{a_{0}}^{1+2 s}} \frac{1}{|y|^{1+2 s}} .
$$

Since $\mu_{a_{0}}=\left(a_{0} V^{\prime \prime}(0)\right)^{-1 / 2 s}$ and $V^{\prime \prime}(0)=2 A$, we obtain the estimate in $(6.27)$. 


\section{Decay improvement of the SOlution LAYER}

Here we use the auxiliary transition layer constructed in Section 6 in order to control the standard transition layer of our problem up to the desired order of approximation. For this we need the following comparison principle, which can be seen as a refinement of the one already given in Proposition 4.1:

Corollary 7.1. Let $v \in L^{\infty}(\mathbb{R}) \cap C^{2}(\mathbb{R})$ such that

$$
\lim _{x \rightarrow \pm \infty} v(x)=0 .
$$

Suppose that there exists a function $d \in L^{\infty}(\mathbb{R})$ such that $d(x) \geqslant \delta>0$ for any $x \in$ $\mathbb{R}$ and for some $\delta>0$, and

$$
L_{s} v=d v+M,
$$

where $M$ is a function that satisfies the following estimate

$$
|M(x)| \leqslant \frac{C}{1+|x|^{1+2 s}} \quad \text { for any } x \in \mathbb{R},
$$

for some constant $C \geqslant 0$.

Then, there exists a constant $\bar{C} \geqslant 0$, depending on $C, d, s$ and $A$ (defined in (6.1)) such that

$$
|v(x)| \leqslant \frac{\bar{C}}{1+|x|^{1+2 s}} \quad \text { for any } x \in \mathbb{R} .
$$

Proof. We consider the function $\phi_{a}$ defined in (6.22) (later we will choose a suitable value of $a$ ). From (6.2), (6.3) and the hypothesis on $M$ in (7.2), we have that there exists a positive constant $K_{a}$ (depending on $a, s, A$ ) such that

$$
|M(x)| \leqslant K_{a} \phi_{a}^{\prime}(x) \quad \text { for any } x \in \mathbb{R} \text {. }
$$

Now, we set $w:=v+c \phi_{a}^{\prime}$, for some positive constant $c$ that will be specified later. From (6.23) and (6.24) we deduce that

$$
a L_{s} \phi_{a}^{\prime}=a V_{a}^{\prime \prime}\left(\phi_{a}\right) \phi_{a}^{\prime}=\frac{V_{a}^{\prime \prime}\left(\phi_{a}\right)}{V_{a}^{\prime \prime}(0)} \phi_{a}^{\prime}=\frac{V^{\prime \prime}\left(\phi_{a}\right)}{V^{\prime \prime}(0)} \phi_{a}^{\prime} .
$$

Therefore, from the properties of the potential $V$ given in Proposition 6.2, we obtain that

$$
\left|a L_{s} \phi_{a}^{\prime}\right| \leqslant C_{V} \phi_{a}^{\prime}
$$

for some constant $C_{V}>0$ depending on $V$. Using (7.4), (7.1) and (7.3), we obtain

$$
\begin{aligned}
L_{s} w-d w & =L_{s} v+c L_{s} \phi_{a}^{\prime}-d v-c d \phi_{a}^{\prime} \\
& \leqslant L_{s} v-d v+\frac{c C_{V}}{a} \phi_{a}^{\prime}-c d \phi_{a}^{\prime} \\
& =M+c\left(\frac{C_{V}}{a}-d\right) \phi_{a}^{\prime} \\
& \leqslant K_{a} \phi_{a}^{\prime}+c\left(\frac{C_{V}}{a}-d\right) \phi_{a}^{\prime} .
\end{aligned}
$$


Now, we choose $\bar{a}$ such that $\frac{C_{V}}{\bar{a}}-d=-\frac{d}{2}$, that is $\bar{a}=\frac{2 C_{V}}{d}$. Hence,

$$
L_{s} w-d w \leqslant\left(K_{\bar{a}}-\frac{c d}{2}\right) \phi_{\bar{a}}^{\prime} .
$$

Finally, we choose $c=\frac{2 K_{\bar{a}}}{d}$ in such a way that $K_{\bar{a}}-\frac{c d}{2}=0$, and therefore

$$
L_{s} w-d w \leqslant 0 .
$$

Since $w$ satisfies the hypotheses of Proposition 4.1, we deduce from it that $w \geqslant 0$, and so

$$
v \geqslant-\frac{2 K_{\bar{a}}}{d} \phi_{\bar{a}}^{\prime}
$$

By similar computation for the function $w:=v-c \phi_{\bar{a}}^{\prime}$, we obtain that $L_{s} w-d w \geqslant 0$, which again, by Proposition 4.1, implies that $w \leqslant 0$, and hence

$$
v \leqslant \frac{2 K_{\bar{a}}}{d} \phi_{\bar{a}}^{\prime}
$$

Putting together (7.5) and (7.6) we obtain that $|v| \leqslant \frac{2 K_{\bar{a}}}{d} \phi_{\bar{a}}^{\prime}$, which implies the desired result thanks to (6.3).

Now, we improve the asymptotics for the layer solution $u$ given in (5.1).

Proposition 7.2. Let $u$ be the solution to (1.5). Then, the following estimate holds:

$$
\left|u(x)-H(x)+\frac{a_{0}}{2 s} \frac{x}{|x|^{1+2 s}}\right| \leqslant \frac{C}{|x|^{1+2 s}} \quad \text { for any } x \in \mathbb{R},
$$

where $a_{0}$ is a positive number such that (6.25) holds, and $C$ is a positive constant depending on $s, a_{0}$ and $A$ (defined in (6.1)).

Proof. We consider the function $\phi_{a_{0}}$ given by (6.22) and such that (6.25) is satisfied. From Proposition 6.4 we know that $\phi_{a_{0}}$ satisfies estimate (6.27).

We define the function $v:=u-\phi_{a_{0}}$. We have that $v \in L^{\infty}(\mathbb{R}) \cap C^{2}(\mathbb{R})$ and

$$
\lim _{x \rightarrow \pm \infty} v(x)=0 \text {. }
$$

Moreover, $v$ satisfies

$$
\begin{aligned}
L_{s} v & =L_{s} u-L_{s} \phi_{a_{0}}=W^{\prime}(u)-V_{a_{0}}^{\prime}\left(\phi_{a_{0}}\right) \\
& =W^{\prime}(u)-W^{\prime}\left(\phi_{a_{0}}\right)+W^{\prime}\left(\phi_{a_{0}}\right)-V_{a_{0}}^{\prime}\left(\phi_{a_{0}}\right) .
\end{aligned}
$$

Now we claim that

$$
W^{\prime}(u)-W^{\prime}\left(\phi_{a_{0}}\right)+W^{\prime}\left(\phi_{a_{0}}\right)-V_{a_{0}}^{\prime}\left(\phi_{a_{0}}\right)=W^{\prime \prime}(0) v+O\left(\frac{1}{1+|x|^{4 s}}\right) .
$$

The proof uses the growth estimates (5.1) and (6.26), by distinguishing the cases in which $x<0$ (hence $H(x)=0$ ) and $x>0$ (hence $H(x)=1$ ). To start, notice that, if $x<0$, we can expand $W^{\prime}(u)$ and $W^{\prime}\left(\phi_{a_{0}}\right)$ in the vicinity of 0 (that is $H(x)=0$ ), recall (5.1) and (6.26), and obtain

$$
W^{\prime}(u)-W^{\prime}\left(\phi_{a_{0}}\right)=W^{\prime \prime}(0)\left(u-\phi_{a_{0}}\right)+O\left(\frac{1}{1+|x|^{4 s}}\right) .
$$

If $x>0$, we can expand $W^{\prime}(u)$ and $W^{\prime}\left(\phi_{a_{0}}\right)$ in the vicinity of 1 and obtain the same result. 
Similarly, when $x<0$, using (6.26) we obtain

$$
\begin{aligned}
W^{\prime}\left(\phi_{a_{0}}\right) & -V_{a_{0}}^{\prime}\left(\phi_{a_{0}}\right) \\
& =W^{\prime}(0)+W^{\prime \prime}(0) \phi_{a_{0}}-V_{a_{0}}^{\prime}(0)-V_{a_{0}}^{\prime \prime}(0) \phi_{a_{0}}+O\left(\frac{1}{1+|x|^{4 s}}\right) \\
& =O\left(\frac{1}{1+|x|^{4 s}}\right),
\end{aligned}
$$

since $W^{\prime}(0)=0=V_{a_{0}}^{\prime}(0)$ (see Proposition $\left.6.2, i\right)$ ) and $W^{\prime \prime}(0)=V_{a_{0}}^{\prime \prime}(0)$ by $(6.25)$. Similarly, if $x>0$, we obtain the same result by expanding $V^{\prime}$ and $W^{\prime}$ in the vicinity of 1 , and so (7.8) follows by collecting the estimates above.

Therefore, by (7.7) and (7.8), we have that the function $v$ satisfies

$$
L_{s} v=W^{\prime \prime}(0) v+M,
$$

where $W^{\prime \prime}(0)>0$ thanks to the hypotheses on $W$ in (1.2), and $M$ is a function that satisfies (7.2). Hence, we can apply Corollary 7.1, thus obtaining that

$$
|v(x)| \leqslant \frac{\bar{C}}{1+|x|^{1+2 s}}
$$

and so

$$
\left|u(x)-\phi_{a_{0}}(x)\right| \leqslant \frac{\bar{C}}{1+|x|^{1+2 s}} .
$$

Putting together (7.9) and (6.27) we obtain the desired estimate.

\section{Proof of Theorem 1.1}

The completion of the proof of Theorem 1.1 uses a system of sub and supersolutions close in spirit to the one in [11]: nevertheless it is necessary in our case to keep track of the different scaling factor induced by the operator $L_{s}$, which produces different orders of $\epsilon$ in the expansions.

In order to prove Theorem 1.1 we consider the solution $\left(x_{i}(t)\right)_{i=1, \ldots, N}$ to the system

$$
\left\{\begin{array}{l}
\dot{x_{i}}=\gamma\left(-\sigma\left(t, x_{i}\right)+\sum_{j \neq i} \frac{x_{i}-x_{j}}{2 s\left|x_{i}-x_{j}\right|^{1+2 s}}\right) \text { in }(0,+\infty), \\
x_{i}(0)=x_{i}^{0},
\end{array}\right.
$$

where $\gamma$ is given in (1.9) and $x_{i}^{0}$ are given for any $i=1, \ldots, N$. Indeed, from [9], we have that there exists a unique solution ${ }^{6}$ to (8.1).

\footnotetext{
${ }^{6}$ More precisely, the existence and uniqueness of solution for (8.1) follows from the classical ODE results once a lower bound on the mutual distance is obtained. Such bound is given in Lemma 8.2 of [9], according to which

$$
\left|x_{i}(t)-x_{j}(t)\right| \geqslant d_{0} e^{-C t},
$$

for any $i \neq j$, where $d_{0}$ is the minimal initial distance and $C>0$ depends on $\gamma$ and $\sigma$.

As a technical remark, we observe that the proof of Lemma 8.2 of [9] does not make use of Assumption (H4) on page 792 there, and therefore we can apply such result in the present framework. Moreover, once the mutual distance is bounded from below, we easily obtain bounds for $\dot{x}_{i}$ and $\ddot{x}_{i}$.
} 
8.1. Construction of sub and supersolutions. To prove existence and uniqueness of the solution to (1.4) we construct suitable sub and supersolutions. We consider an auxiliary ${ }^{7}$ parameter $\delta>0$ and define $\left(\bar{x}_{i}(t)\right)_{i=1, \ldots, N}$ to be the solution of the system

$$
\left\{\begin{array}{l}
\dot{\bar{x}}_{i}=\gamma\left(-\delta-\sigma\left(t, \bar{x}_{i}\right)+\sum_{j \neq i} \frac{\bar{x}_{i}-\bar{x}_{j}}{2 s\left|\bar{x}_{i}-\bar{x}_{j}\right|^{1+2 s}}\right) \text { in }(0,+\infty), \\
\bar{x}_{i}(0)=x_{i}^{0}-\delta
\end{array}\right.
$$

Moreover, we set

$$
\bar{c}_{i}(t):=\dot{\bar{x}}_{i}(t)
$$

and

$$
\tilde{\sigma}:=\frac{\delta+\sigma}{\beta}, \quad \text { where } \beta=W^{\prime \prime}(0) \text { was introduced in (1.11). }
$$

We also define

$$
\bar{v}_{\epsilon}(t, x):=\epsilon^{2 s} \tilde{\sigma}(t, x)+\sum_{i=1}^{N}\left\{u\left(\frac{x-\bar{x}_{i}(t)}{\epsilon}\right)-\epsilon^{2 s} \bar{c}_{i}(t) \psi\left(\frac{x-\bar{x}_{i}(t)}{\epsilon}\right)\right\},
$$

where $u$ is given in Theorem 5.1 and $\psi$ in Theorem 5.2. The asymptotics of $\bar{v}_{\epsilon}(t, x)$ is compatible with the convex envelope behavior in (1.13), according to the following observation:

Lemma 8.1. For any $(t, x) \in[0, T) \times \mathbb{R}$, we have that

$$
\lim _{\delta \rightarrow 0^{+}} \limsup _{\substack{\left(t^{\prime}, x^{\prime}\right) \rightarrow(t, x) \\ \epsilon \rightarrow 0}} \bar{v}_{\epsilon}\left(t^{\prime}, x^{\prime}\right) \leqslant\left(v_{0}\right)^{*}(t, x) .
$$

Proof. We define $H^{*}(x):=H(x)$ for any $x \neq 0$, and $H^{*}(0):=1$. We point out that

$$
H \leqslant H^{*} \text { and } \limsup _{r^{\prime} \rightarrow r} H^{*}\left(r^{\prime}\right) \leqslant H^{*}(r) .
$$

Now we prove that

$$
\sum_{i=1}^{N} H^{*}\left(x-x_{i}(t)\right)=\left(v_{0}\right)^{*}(t, x) .
$$

For this, we use the definitions in (1.7) and (1.12) to write

$$
\left(v_{0}\right)^{*}(t, x)=\limsup _{\left(t^{\prime}, x^{\prime}\right) \rightarrow(t, x)} \sum_{i=1}^{N} H\left(x^{\prime}-x_{i}\left(t^{\prime}\right)\right) .
$$

Moreover, we observe that if $x$ and $t$ are such that $x-x_{i}(t) \neq 0$ for any $i \in$ $\{1, \ldots, N\}$, then (8.7) is obvious by the continuity of the functions. So, we may suppose that $x-x_{i_{0}}(t)=0$ for some $i_{0}$. Since the $x_{i}(t)$ are separated, this implies

\footnotetext{
7 We will fix $\delta>0$ and suppose that $\epsilon$ is small also possibly in dependence of $\delta$. At the end, after having performed the limit in $\epsilon$, we will have the freedom of taking $\delta$ as small as we wish, see Lemma 8.1 for this.
} 
that $x-x_{i}(t) \neq 0$ for any $i \neq i_{0}$ hence continuity holds when $i \neq i_{0}$. That is, (8.8) gives that

$$
\begin{aligned}
\left(v_{0}\right)^{*}(t, x) & =\limsup _{\left(t^{\prime}, x^{\prime}\right) \rightarrow(t, x)} H\left(x^{\prime}-x_{i_{0}}\left(t^{\prime}\right)\right)+\sum_{i \neq i_{0}} H\left(x-x_{i}(t)\right) \\
& =\limsup _{\left(t^{\prime}, x^{\prime}\right) \rightarrow(t, x)} H\left(x^{\prime}-x_{i_{0}}\left(t^{\prime}\right)\right)+\sum_{i \neq i_{0}} H^{*}\left(x-x_{i}(t)\right) .
\end{aligned}
$$

Now we specialize a sequence, by choosing $x^{\prime}=\tilde{x}_{n}:=x+e^{-n}$ and $t^{\prime}=\tilde{t}_{n}:=t$. In this way,

$$
\tilde{x}_{n}-x_{i_{0}}\left(\tilde{t}_{n}\right)=x-x_{i_{0}}(t)+e^{-n}=e^{-n}>0
$$

hence

$$
H\left(\tilde{x}_{n}-x_{i_{0}}\left(\tilde{t}_{n}\right)\right)=1=H^{*}(0)=H^{*}\left(x-x_{i_{0}}(t)\right)
$$

and so

$$
\begin{aligned}
\limsup _{\left(t^{\prime}, x^{\prime}\right) \rightarrow(t, x)} H\left(x^{\prime}-x_{i_{0}}\left(t^{\prime}\right)\right) & \geqslant \limsup _{n \rightarrow+\infty} H\left(\tilde{x}_{n}-x_{i_{0}}\left(\tilde{t}_{n}\right)\right) \\
& =H^{*}\left(x-x_{i_{0}}(t)\right) .
\end{aligned}
$$

By plugging this into (8.9), we obtain

$$
\left(v_{0}\right)^{*}(t, x) \geqslant H^{*}\left(x-x_{i_{0}}(t)\right)+\sum_{i \neq i_{0}} H^{*}\left(x-x_{i}(t)\right)=\sum_{i=1}^{N} H^{*}\left(x-x_{i}(t)\right) .
$$

On the other hand, from (8.8) and (8.6),

$$
\begin{aligned}
\left(v_{0}\right)^{*}(t, x) & \leqslant \sum_{i=1}^{N} \limsup _{\left(t^{\prime}, x^{\prime}\right) \rightarrow(t, x)} H\left(x^{\prime}-x_{i}\left(t^{\prime}\right)\right) \\
& \leqslant \sum_{i=1}^{N} \limsup _{\left(t^{\prime}, x^{\prime}\right) \rightarrow(t, x)} H^{*}\left(x^{\prime}-x_{i}\left(t^{\prime}\right)\right) \\
& \leqslant \sum_{i=1}^{N} H^{*}\left(x-x_{i}(t)\right) .
\end{aligned}
$$

By collecting (8.10) and (8.11), we complete the proof of (8.7).

Now we notice that

$$
\limsup _{\substack{\left(t^{\prime}, x^{\prime}\right) \rightarrow(t, x) \\ \epsilon \rightarrow 0}} \bar{v}_{\epsilon}\left(t^{\prime}, x^{\prime}\right)=\limsup _{\substack{\left(t^{\prime}, x^{\prime}\right) \rightarrow(t, x) \\ \epsilon \rightarrow 0}} \sum_{i=1}^{N} u\left(\frac{x^{\prime}-\bar{x}_{i}\left(t^{\prime}\right)}{\epsilon}\right),
$$

since the other terms in (8.5) vanish with $\epsilon$. Fixed $x$ and $t$, we let $i_{0}$ such that

$$
x \in\left[\bar{x}_{i_{0}}(t), \bar{x}_{i_{0}+1}(t)\right)
$$

and we define

$$
\vartheta:=\min _{i \neq i_{0}}\left|x-\bar{x}_{i}(t)\right| .
$$

By construction $\vartheta>0$ and we will be free to suppose that $\epsilon,\left|t^{\prime}-t\right|$ and $\left|x^{\prime}-x\right|$ are much smaller than $\theta$.

Now we define

$$
x^{\prime \prime}=x^{\prime}+\left|x^{\prime}-x\right|+\sqrt{\epsilon}+\sqrt{\left|t^{\prime}-t\right|} .
$$


Notice that $x^{\prime \prime}$ is a sequence constructed from the original sequence $\left(t^{\prime}, x^{\prime}, \epsilon\right) \rightarrow$ $(t, x, 0)$ therefore $x^{\prime \prime}$ approaches $x$. Since $u$ is increasing, we have that

$$
u\left(\frac{x^{\prime}-\bar{x}_{i}\left(t^{\prime}\right)}{\epsilon}\right) \leqslant u\left(\frac{x^{\prime \prime}-\bar{x}_{i}\left(t^{\prime}\right)}{\epsilon}\right) .
$$

More precisely, from (8.14) and (8.13),

$$
\begin{aligned}
x^{\prime \prime}-\bar{x}_{i_{0}}(t) & =\left(x^{\prime}-x\right)+x-\bar{x}_{i_{0}}(t)+\left|x^{\prime}-x\right|+\sqrt{\epsilon}+\sqrt{\left|t^{\prime}-t\right|} \\
& \geqslant x-\bar{x}_{i_{0}}(t)+\sqrt{\epsilon}+\sqrt{\left|t^{\prime}-t\right|} \\
& \geqslant \sqrt{\epsilon}+\sqrt{\left|t^{\prime}-t\right|} .
\end{aligned}
$$

Furthermore, if $i \neq i_{0}$,

$$
\begin{aligned}
\left|\bar{x}_{i}(t)-x^{\prime \prime}\right| & =\left|\bar{x}_{i}(t)-x+\left(x-x^{\prime}\right)-\right| x^{\prime}-x\left|-\sqrt{\epsilon}-\sqrt{\left|t^{\prime}-t\right|}\right| \\
& \geqslant \theta-2\left|x^{\prime}-x\right|-\sqrt{\epsilon}-\sqrt{\left|t^{\prime}-t\right|} \\
& \geqslant \frac{\theta}{2} .
\end{aligned}
$$

This and (8.16) imply that, for every $i \in\{1, \ldots, N\}$,

$$
\left|x^{\prime \prime}-\bar{x}_{i}(t)\right| \geqslant \sqrt{\epsilon}+\sqrt{\left|t^{\prime}-t\right|} .
$$

By the regularity theory for ODEs, we have that

$$
\left|\bar{x}_{i}\left(t^{\prime}\right)-\bar{x}_{i}(t)\right|=O\left(\left|t^{\prime}-t\right|\right)
$$

hence (8.17) gives that

$$
\left|x^{\prime \prime}-\bar{x}_{i}\left(t^{\prime}\right)\right| \geqslant \sqrt{\epsilon}+\sqrt{\left|t^{\prime}-t\right|}-O\left(\left|t^{\prime}-t\right|\right) \geqslant \sqrt{\epsilon},
$$

since $\left|t^{\prime}-t\right|$ is an infinitesimal sequence. As a consequence of this, we have that

$$
\left|\frac{x^{\prime \prime}-\bar{x}_{i}\left(t^{\prime}\right)}{\epsilon}\right| \geqslant \frac{1}{\sqrt{\epsilon}},
$$

that is a diverging sequence. Therefore, recalling (5.1), and observing that $H(x / \epsilon)=$ $H(x)$ for any $x \in \mathbb{R}$, since $\epsilon>0$, we obtain

$$
\begin{aligned}
& \limsup _{\substack{\left(t^{\prime}, x^{\prime}\right) \rightarrow(t, x) \\
\epsilon \rightarrow 0}} u\left(\frac{x^{\prime \prime}-\bar{x}_{i}\left(t^{\prime}\right)}{\epsilon}\right)-H\left(x^{\prime \prime}-\bar{x}_{i}\left(t^{\prime}\right)\right) \\
& \quad=\limsup _{\substack{\left(t^{\prime}, x^{\prime}\right) \rightarrow(t, x) \\
\epsilon \rightarrow 0}} u\left(\frac{x^{\prime \prime}-\bar{x}_{i}\left(t^{\prime}\right)}{\epsilon}\right)-H\left(\frac{x^{\prime \prime}-\bar{x}_{i}\left(t^{\prime}\right)}{\epsilon}\right)=0 .
\end{aligned}
$$

Using this, (8.12) and (8.15) we obtain

$$
\begin{aligned}
\limsup _{\substack{\left(t^{\prime}, x^{\prime}\right) \rightarrow(t, x) \\
\epsilon \rightarrow 0}} \bar{v}_{\epsilon}\left(t^{\prime}, x^{\prime}\right) & \leqslant \limsup _{\substack{\left(t^{\prime}, x^{\prime}\right) \rightarrow(t, x) \\
\epsilon \rightarrow 0}} \sum_{i=1}^{N} u\left(\frac{x^{\prime \prime}-\bar{x}_{i}\left(t^{\prime}\right)}{\epsilon}\right) \\
& \leqslant \sum_{i=1}^{N} \limsup _{\substack{\left(t^{\prime}, x^{\prime}\right) \rightarrow(t, x) \\
\epsilon \rightarrow 0}} u\left(\frac{x^{\prime \prime}-\bar{x}_{i}\left(t^{\prime}\right)}{\epsilon}\right) \\
& \leqslant \sum_{i=1}^{N} \limsup _{\substack{\left(t^{\prime}, x^{\prime}\right) \rightarrow(t, x) \\
\epsilon \rightarrow 0}} H\left(x^{\prime \prime}-\bar{x}_{i}\left(t^{\prime}\right)\right) .
\end{aligned}
$$


Now we use the ODE theory to notice that $\left|\bar{x}_{i}(t)-x_{i}(t)\right| \leqslant O(\delta)$, since both the forcing term and the initial data of the two equations are $\delta$-close to each other (compare (1.8) and (8.2)). In particular

$$
x^{\prime \prime}-\bar{x}_{i}\left(t^{\prime}\right) \leqslant x^{\prime \prime}-x_{i}\left(t^{\prime}\right)+O(\delta)
$$

and so, using the fact that $H$ is monotone,

$$
H\left(x^{\prime \prime}-\bar{x}_{i}\left(t^{\prime}\right)\right) \leqslant H\left(x^{\prime \prime}-x_{i}\left(t^{\prime}\right)+O(\delta)\right) .
$$

This, (8.18) and (8.6) imply that

$$
\begin{aligned}
\lim _{\delta \rightarrow 0^{+}} \limsup _{\substack{\left(t^{\prime}, x^{\prime}\right) \rightarrow(t, x) \\
\epsilon \rightarrow 0}} \bar{v}_{\epsilon}\left(t^{\prime}, x^{\prime}\right) \leqslant \lim _{\delta \rightarrow 0^{+}} \sum_{i=1}^{N} \limsup _{\substack{\left(t^{\prime}, x^{\prime}\right) \rightarrow(t, x) \\
\epsilon \rightarrow 0}} H\left(x^{\prime \prime}-x_{i}\left(t^{\prime}\right)+O(\delta)\right) \\
\leqslant \lim _{\delta \rightarrow 0^{+}} \sum_{i=1}^{N} \limsup _{\substack{\left(t^{\prime}, x^{\prime}\right) \rightarrow(t, x) \\
\epsilon \rightarrow 0}} H^{*}\left(x^{\prime \prime}-x_{i}\left(t^{\prime}\right)+O(\delta)\right) \\
\leqslant \lim _{\delta \rightarrow 0^{+}} \sum_{i=1}^{N} H^{*}\left(x-x_{i}(t)+O(\delta)\right) \\
\leqslant \sum_{i=1}^{N} H^{*}\left(x-x_{i}(t)\right) .
\end{aligned}
$$

The desired result then follows from (8.7).

In the following proposition, we control the initial condition of $\bar{v}_{\epsilon}$ :

Proposition 8.2. Assume that (1.2), (1.3) and (1.10) hold. Then, there exists $\delta_{0}>$ 0 such that, for every $0<\delta \leqslant \delta_{0}$, one has

$$
\bar{v}_{\epsilon}(0, x) \geqslant v_{\epsilon}^{0}(x) \quad \text { for any } x \in \mathbb{R},
$$

for any $\epsilon>0$ sufficiently small, where $v_{\epsilon}^{0}$ is defined in (1.10).

Proof. We choose

$$
0<\delta<\min _{i=1, \ldots, N-1}\left(x_{i+1}^{0}-x_{i}^{0}\right) .
$$

In this way we have that

$$
x_{i}^{0}<\bar{x}_{i+1}(0)<x_{i+1}^{0} \quad \text { for any } i=1, \ldots, N-1 .
$$

As a consequence of this and of the fact that $u$ is increasing, we have that

$$
u\left(\frac{x-\bar{x}_{i}(0)}{\epsilon}\right) \geqslant u\left(\frac{x-x_{i}^{0}}{\epsilon}\right) .
$$

Also, using the fact that

$$
\lim _{x \rightarrow \pm \infty} \psi(x)=0
$$

due to (5.2), we can choose $R>0$ so large that

$$
\frac{\delta}{\beta} \geqslant\left(\sum_{i=1}^{N}\left|\bar{c}_{i}(0)\right|\right) \sup _{\mathbb{R} \backslash(-R, R)}|\psi| .
$$


Now, in order to prove (8.19), we distinguish the points $x$ that are very close to $\bar{x}_{i}(0)$ for some $i \in\{1, \ldots, N\}$ and the points that are sufficiently separated. For this, suppose first that

$$
\left|x-\bar{x}_{i}(0)\right| \geqslant \epsilon R \quad \text { for every } i=1, \ldots, N .
$$

Then $\left(x-\bar{x}_{i}(0)\right) / \epsilon \in \mathbb{R} \backslash(-R, R)$ and so, by $(8.21)$,

$$
\left|\sum_{i=1}^{N} \bar{c}_{i}(0) \psi\left(\frac{x-\bar{x}_{i}(0)}{\epsilon}\right)\right| \leqslant \frac{\delta}{\beta} .
$$

So, recalling (8.5), (8.20), (8.4) and (1.10),

$$
\begin{aligned}
\bar{v}_{\epsilon}(0, x) & =\epsilon^{2 s} \tilde{\sigma}(0, x)+\sum_{i=1}^{N}\left\{u\left(\frac{x-\bar{x}_{i}(0)}{\epsilon}\right)-\epsilon^{2 s} \bar{c}_{i}(0) \psi\left(\frac{x-\bar{x}_{i}(0)}{\epsilon}\right)\right\} \\
& \geqslant \frac{\epsilon^{2 s}}{\beta}(\delta+\sigma(0, x))+\sum_{i=1}^{N}\left\{u\left(\frac{x-x_{i}^{0}}{\epsilon}\right)-\epsilon^{2 s} \bar{c}_{i}(0) \psi\left(\frac{x-\bar{x}_{i}(0)}{\epsilon}\right)\right\} \\
& =v_{\epsilon}^{0}(x)+\epsilon^{2 s}\left(\frac{\delta}{\beta}-\sum_{i=1}^{N} \bar{c}_{i}(0) \psi\left(\frac{x-\bar{x}_{i}(0)}{\epsilon}\right)\right)
\end{aligned}
$$

which, together with (8.23), implies that

$$
\bar{v}_{\epsilon}(0, x) \geqslant v_{\epsilon}^{0}(x) .
$$

This proves (8.19) in case (8.22) holds true.

Now, conversely, suppose that there exists an index $i_{0} \in\{1, \ldots, N\}$ such that $\left|x-\bar{x}_{i_{0}}(0)\right|<\epsilon R$. Then, in this case, the fact that $u$ is increasing implies that

$$
u\left(\frac{x-\bar{x}_{i_{0}}(0)}{\epsilon}\right) \geqslant u(-R)>0,
$$

and

$$
u\left(\frac{x-x_{i_{0}}^{0}}{\epsilon}\right)=u\left(\frac{x-\bar{x}_{i_{0}}(0)-\delta}{\epsilon}\right) \leqslant u\left(R-\frac{\delta}{\epsilon}\right) .
$$

Hence, from (8.25) and (5.1),

$$
u\left(\frac{x-x_{i_{0}}^{0}}{\epsilon}\right) \leqslant\left(\frac{C \epsilon}{\delta}\right)^{2 s}
$$

for some $C>0$, when $\epsilon$ is small enough. Using this and (8.24) we conclude that, for $\epsilon$ sufficiently small,

$$
\begin{aligned}
u\left(\frac{x-\bar{x}_{i_{0}}(0)}{\epsilon}\right) & \geqslant u(-R) \\
& \geqslant \frac{u(-R)}{2}+\left(\frac{C \epsilon}{\delta}\right)^{2 s}+\epsilon^{2 s}\|\psi\|_{L^{\infty}(\mathbb{R})} \sum_{i=1}^{N}\left|\bar{c}_{i}(0)\right| \\
& \geqslant u\left(\frac{x-x_{i_{0}}^{0}}{\epsilon}\right)+\sum_{i=1}^{N} \epsilon^{2 s} \bar{c}_{i}(0) \psi\left(\frac{x-\bar{x}_{i}(0)}{\epsilon}\right) .
\end{aligned}
$$


Hence, using this estimate and (8.20),

$$
\begin{aligned}
& \bar{v}_{\epsilon}(0, x) \\
= & \epsilon^{2 s} \tilde{\sigma}(0, x)+\sum_{i=1}^{N}\left\{u\left(\frac{x-\bar{x}_{i}(0)}{\epsilon}\right)-\epsilon^{2 s} \bar{c}_{i}(0) \psi\left(\frac{x-\bar{x}_{i}(0)}{\epsilon}\right)\right\} \\
= & \frac{\epsilon^{2 s}}{\beta}(\delta+\sigma(0, x))+\sum_{\substack{1 \leqslant i \leqslant N \\
i \neq i_{0}}} u\left(\frac{x-\bar{x}_{i}(0)}{\epsilon}\right)+u\left(\frac{x-\bar{x}_{i_{0}}(0)}{\epsilon}\right)-\sum_{i=1}^{N} \epsilon^{2 s} \bar{c}_{i}(0) \psi\left(\frac{x-\bar{x}_{i}(0)}{\epsilon}\right) \\
\geqslant & \frac{\epsilon^{2 s}}{\beta}(\delta+\sigma(0, x))+\sum_{\substack{1 \leqslant i \leqslant N \\
i \neq i_{0}}} u\left(\frac{x-x_{i}^{0}}{\epsilon}\right)+u\left(\frac{x-x_{i_{0}}^{0}}{\epsilon}\right) \\
= & \frac{\epsilon^{2 s}}{\beta}(\delta+\sigma(0, x))+\sum_{\substack{i=1 \\
\beta}} u\left(\frac{x-x_{i}^{0}}{\epsilon}\right) \\
= & \frac{\epsilon^{2 s} \delta}{\beta}+v_{\epsilon}^{0}(x) \\
\geqslant & v_{\epsilon}^{0}(x) .
\end{aligned}
$$

This concludes the proof of (8.19) in this case too.

Now, we set

$$
\tilde{u}_{i}:=u\left(\frac{x-\bar{x}_{i}(t)}{\epsilon}\right)-H\left(\frac{x-\bar{x}_{i}(t)}{\epsilon}\right),
$$

where $H$ is the Heaviside function, and

$$
\psi_{i}:=\psi\left(\frac{x-\bar{x}_{i}(t)}{\epsilon}\right)
$$

Let also

$$
I_{\epsilon}:=\epsilon\left(\bar{v}_{\epsilon}\right)_{t}+\frac{1}{\epsilon^{2 s}}\left(W^{\prime}\left(\bar{v}_{\epsilon}\right)-\epsilon^{2 s} L_{s} \bar{v}_{\epsilon}-\epsilon^{2 s} \sigma\right) .
$$

Our goal is to estimate $I_{\epsilon}$ for small $\epsilon$. Functions that are bounded by $\tilde{u}_{i}$, or $\psi_{i}$, up to multiplicative constants, will be denoted by $O\left(\tilde{u}_{i}\right)$, or $O\left(\psi_{i}\right)$, respectively. The reason for this notation is that, when $x \neq \bar{x}_{i}(t)$, both $\tilde{u}_{i}$ and $\psi_{i}$ are infinitesimal for small $\epsilon$, in the light of (5.1) and (5.2). With this setting, we have the following estimate:

Lemma 8.3. Assume that (1.2), (1.3) and (1.10) hold. Then, for every $i_{0} \in$ $\{1, \ldots, N\}$, we have

$$
I_{\epsilon}=e_{\epsilon}^{i_{0}}+(\beta \tilde{\sigma}-\sigma)+O\left(\tilde{u}_{i_{0}}\right)\left(\eta \bar{c}_{i_{0}}+\tilde{\sigma}+\sum_{\substack{1 \leqslant i \leqslant N \\ i \neq i_{0}}} \frac{\tilde{u}_{i}}{\epsilon^{2 s}}\right),
$$

where $\beta$ is given in (1.11), $\tilde{\sigma}$ in (8.4), $\eta$ in (5.3), and the error $e_{\epsilon}^{i_{0}}$ is given by

$$
e_{\epsilon}^{i_{0}}:=O\left(\epsilon^{2 s}\right)+\sum_{\substack{1 \leqslant i \leqslant N \\ i \neq i_{0}}} O\left(\psi_{i}\right)+\sum_{\substack{1 \leqslant i \leqslant N \\ i \neq i_{0}}} O\left(\tilde{u}_{i}\right)+\sum_{\substack{1 \leqslant i \leqslant N \\ i \neq i_{0}}} O\left(\frac{\tilde{u}_{i}^{2}}{\epsilon^{2 s}}\right) .
$$


Proof. We compute $I_{\epsilon}$ piece by piece. First of all, by differentiating (8.5) we obtain

$$
\begin{aligned}
\left(\bar{v}_{\epsilon}\right)_{t} & =O\left(\epsilon^{2 s}\right)+\sum_{i=1}^{N} \frac{\partial}{\partial t} u\left(\frac{x-\bar{x}_{i}(t)}{\epsilon}\right)-\epsilon^{2 s} \sum_{i=1}^{N} \frac{\partial}{\partial t}\left[\bar{c}_{i}(t) \psi\left(\frac{x-\bar{x}_{i}(t)}{\epsilon}\right)\right] \\
& =O\left(\epsilon^{2 s}\right)-\sum_{i=1}^{N} \frac{\dot{x}_{i}(t)}{\epsilon} u^{\prime}\left(\frac{x-\bar{x}_{i}(t)}{\epsilon}\right)-\epsilon^{2 s} \sum_{i=1}^{N}\left[\dot{\bar{c}}_{i}(t) \psi\left(\frac{x-\bar{x}_{i}(t)}{\epsilon}\right)-\frac{\dot{\bar{x}}_{i}(t)}{\epsilon} \bar{c}_{i}(t) \psi^{\prime}\left(\frac{x-\bar{x}_{i}(t)}{\epsilon}\right)\right] .
\end{aligned}
$$

That is, from (8.3), multiplying by $\epsilon$ and dropping the explicit dependence on $t$ for short, we obtain

$$
\epsilon\left(\bar{v}_{\epsilon}\right)_{t}=O\left(\epsilon^{1+2 s}\right)-\sum_{i=1}^{N} \bar{c}_{i} u^{\prime}\left(\frac{x-\bar{x}_{i}}{\epsilon}\right)-\sum_{i=1}^{N} \epsilon^{1+2 s} \dot{\bar{c}}_{i} \psi_{i}+\sum_{i=1}^{N} \epsilon^{2 s} \bar{c}_{i}^{2} \psi^{\prime}\left(\frac{x-\bar{x}_{i}}{\epsilon}\right) .
$$

Now we notice that we can bound $\psi$ and $\psi^{\prime}$ thanks to Theorem 5.2. Moreover, $\dot{\bar{c}}_{i}$ is bounded for every $i=1, \ldots, N$ (recall the bound on $\ddot{x}_{i}$ discussed in the footnote of page 30$)$. Therefore (8.29) reduces to

$$
\epsilon\left(\bar{v}_{\epsilon}\right)_{t}=O\left(\epsilon^{2 s}\right)-\sum_{i=1}^{N} \bar{c}_{i} u^{\prime}\left(\frac{x-\bar{x}_{i}}{\epsilon}\right) .
$$

Also, we use the periodicity of $W$ (to remove the Heaviside function inside the potential) and a Taylor expansion in the vicinity of $\tilde{u}_{i_{0}}$, to calculate:

$$
\begin{aligned}
W^{\prime}\left(\bar{v}_{\epsilon}\right) & =W^{\prime}\left(\epsilon^{2 s} \tilde{\sigma}+\sum_{i=1}^{N} u\left(\frac{x-\bar{x}_{i}}{\epsilon}\right)-\epsilon^{2 s} \sum_{i=1}^{N} \bar{c}_{i} \psi\left(\frac{x-\bar{x}_{i}}{\epsilon}\right)\right) \\
& =W^{\prime}\left(\epsilon^{2 s} \tilde{\sigma}+\sum_{i=1}^{N} \tilde{u}_{i}-\epsilon^{2 s} \sum_{i=1}^{N} \bar{c}_{i} \psi_{i}\right) \\
& =W^{\prime}\left(\tilde{u}_{i_{0}}+\epsilon^{2 s} \tilde{\sigma}+\sum_{\substack{1 \leqslant i \leqslant N \\
i \neq i_{0}}} \tilde{u}_{i}-\epsilon^{2 s} \sum_{i=1}^{N} \bar{c}_{i} \psi_{i}\right) \\
& =W^{\prime}\left(\tilde{u}_{i_{0}}\right)+W^{\prime \prime}\left(\tilde{u}_{i_{0}}\right)\left(\epsilon^{2 s} \tilde{\sigma}+\sum_{\substack{1 \leqslant i \leqslant N \\
i \neq i_{0}}} \tilde{u}_{i}-\epsilon^{2 s} \sum_{i=1}^{N} \bar{c}_{i} \psi_{i}\right)+O\left(\epsilon^{4 s}\right)+\sum_{\substack{1 \leqslant i \leqslant N \\
i \neq i_{0}}} O\left(\tilde{u}_{i}^{2}\right) .
\end{aligned}
$$

Now we recall that $L_{s} \sigma$ is bounded thanks to (1.3). Hence we use the scaling properties of $L_{s},(1.5)$ and (5.2) to evaluate the following expression:

$$
\begin{aligned}
\epsilon^{2 s} L_{s} \bar{v}_{\epsilon} & =\epsilon^{2 s} L_{s}\left(\epsilon^{2 s} \tilde{\sigma}+\sum_{i=1}^{N} u\left(\frac{x-\bar{x}_{i}}{\epsilon}\right)-\epsilon^{2 s} \sum_{i=1}^{N} \bar{c}_{i} \psi\left(\frac{x-\bar{x}_{i}}{\epsilon}\right)\right) \\
& =O\left(\epsilon^{4 s}\right)+\sum_{i=1}^{N} L_{s} u\left(\frac{x-\bar{x}_{i}}{\epsilon}\right)-\epsilon^{2 s} \sum_{i=1}^{N} \bar{c}_{i} L_{s} \psi\left(\frac{x-\bar{x}_{i}}{\epsilon}\right) \\
& =O\left(\epsilon^{4 s}\right)+\sum_{i=1}^{N} W^{\prime}\left(\tilde{u}_{i}\right)-\epsilon^{2 s} \sum_{i=1}^{N} \bar{c}_{i}\left[W^{\prime \prime}\left(\tilde{u}_{i}\right) \psi_{i}+u^{\prime}\left(\frac{x-\bar{x}_{i}}{\epsilon}\right)+\eta\left(W^{\prime \prime}\left(\tilde{u}_{i}\right)-W^{\prime \prime}(0)\right)\right] .
\end{aligned}
$$


So we sum up (8.30), (8.31) and (8.32): recalling (8.27) and noticing that the two terms involving $u^{\prime}$ cancel, we obtain that

$$
\begin{aligned}
I_{\epsilon}= & \epsilon\left(\bar{v}_{\epsilon}\right)_{t}+\epsilon^{-2 s} W^{\prime}\left(\bar{v}_{\epsilon}\right)-L_{s} \bar{v}_{\epsilon}-\sigma \\
= & \epsilon^{-2 s} W^{\prime}\left(\tilde{u}_{i_{0}}\right)+W^{\prime \prime}\left(\tilde{u}_{i_{0}}\right)\left(\tilde{\sigma}+\epsilon^{-2 s} \sum_{i \neq i_{0}} \tilde{u}_{i}-\sum_{1 \leqslant i \leqslant N} \bar{c}_{i} \psi_{i}\right) \\
& -\epsilon^{-2 s} \sum_{1 \leqslant i \leqslant N} W^{\prime}\left(\tilde{u}_{i}\right)+\sum_{1 \leqslant i \leqslant N} \bar{c}_{i}\left[W^{\prime \prime}\left(\tilde{u}_{i}\right) \psi_{i}+\eta\left(W^{\prime \prime}\left(\tilde{u}_{i}\right)-W^{\prime \prime}(0)\right)\right] \\
& -\sigma+O\left(\epsilon^{2 s}\right)+\sum_{i \neq i_{0}} O\left(\epsilon^{-2 s} \tilde{u}_{i}^{2}\right) .
\end{aligned}
$$

Now we collect some terms in the previous expression, namely we observe that

$$
\epsilon^{-2 s} W^{\prime}\left(\tilde{u}_{i_{0}}\right)-\epsilon^{-2 s} \sum_{1 \leqslant i \leqslant N} W^{\prime}\left(\tilde{u}_{i}\right)=-\epsilon^{-2 s} \sum_{i \neq i_{0}} W^{\prime}\left(\tilde{u}_{i}\right)
$$

and that

$$
-W^{\prime \prime}\left(\tilde{u}_{i_{0}}\right) \sum_{1 \leqslant i \leqslant N} \bar{c}_{i} \psi_{i}+\sum_{1 \leqslant i \leqslant N} \bar{c}_{i} W^{\prime \prime}\left(\tilde{u}_{i}\right) \psi_{i}=\sum_{i \neq i_{0}} \bar{c}_{i}\left(W^{\prime \prime}\left(\tilde{u}_{i}\right)-W^{\prime \prime}\left(\tilde{u}_{i_{0}}\right)\right) \psi_{i} .
$$

Therefore we obtain

$$
\begin{aligned}
I_{\epsilon}= & -\epsilon^{-2 s} \sum_{i \neq i_{0}} W^{\prime}\left(\tilde{u}_{i}\right)+\sum_{i \neq i_{0}} \bar{c}_{i}\left(W^{\prime \prime}\left(\tilde{u}_{i}\right)-W^{\prime \prime}\left(\tilde{u}_{i_{0}}\right)\right) \psi_{i} \\
& +W^{\prime \prime}\left(\tilde{u}_{i_{0}}\right)\left(\tilde{\sigma}+\epsilon^{-2 s} \sum_{i \neq i_{0}} \tilde{u}_{i}\right) \\
& +\sum_{1 \leqslant i \leqslant N} \bar{c}_{i} \eta\left(W^{\prime \prime}\left(\tilde{u}_{i}\right)-W^{\prime \prime}(0)\right) \\
& -\sigma+O\left(\epsilon^{2 s}\right)+\sum_{i \neq i_{0}} O\left(\epsilon^{-2 s} \tilde{u}_{i}^{2}\right) .
\end{aligned}
$$

Now, since $W^{\prime}(0)=0$, we use a Taylor expansion around 0 to see that

$$
\epsilon^{-2 s} W^{\prime}\left(\tilde{u}_{i}\right)=\epsilon^{-2 s} W^{\prime \prime}(0) \tilde{u}_{i}+O\left(\epsilon^{-2 s} \tilde{u}_{i}^{2}\right)
$$

so that

$$
\begin{aligned}
I_{\epsilon}= & -\epsilon^{-2 s} W^{\prime \prime}(0) \sum_{i \neq i_{0}} \tilde{u}_{i}+\sum_{i \neq i_{0}} \bar{c}_{i}\left(W^{\prime \prime}\left(\tilde{u}_{i}\right)-W^{\prime \prime}\left(\tilde{u}_{i_{0}}\right)\right) \psi_{i} \\
& +W^{\prime \prime}\left(\tilde{u}_{i_{0}}\right)\left(\tilde{\sigma}+\epsilon^{-2 s} \sum_{i \neq i_{0}} \tilde{u}_{i}\right) \\
& +\sum_{1 \leqslant i \leqslant N} \bar{c}_{i} \eta\left(W^{\prime \prime}\left(\tilde{u}_{i}\right)-W^{\prime \prime}(0)\right) \\
& -\sigma+O\left(\epsilon^{2 s}\right)+\sum_{i \neq i_{0}} O\left(\epsilon^{-2 s} \tilde{u}_{i}^{2}\right) .
\end{aligned}
$$


Now it is convenient to add and subtract the term $W^{\prime \prime}(0) \tilde{\sigma}$ and collect all the terms containing the common factor $\left(W^{\prime \prime}\left(\tilde{u}_{i_{0}}\right)-W^{\prime \prime}(0)\right)$. We get

$$
\begin{aligned}
I_{\epsilon}= & \left(W^{\prime \prime}\left(\tilde{u}_{i_{0}}\right)-W^{\prime \prime}(0)\right) \epsilon^{-2 s} \sum_{i \neq i_{0}} \tilde{u}_{i}+\sum_{i \neq i_{0}} \bar{c}_{i}\left(W^{\prime \prime}\left(\tilde{u}_{i}\right)-W^{\prime \prime}\left(\tilde{u}_{i_{0}}\right)\right) \psi_{i} \\
& +\left(W^{\prime \prime}\left(\tilde{u}_{i_{0}}\right)-W^{\prime \prime}(0)\right) \tilde{\sigma}+W^{\prime \prime}(0) \tilde{\sigma} \\
& +\sum_{i \neq i_{0}} \bar{c}_{i} \eta\left(W^{\prime \prime}\left(\tilde{u}_{i}\right)-W^{\prime \prime}(0)\right)+\bar{c}_{i_{0}} \eta\left(W^{\prime \prime}\left(\tilde{u}_{i_{0}}\right)-W^{\prime \prime}(0)\right) \\
& -\sigma+O\left(\epsilon^{2 s}\right)+\sum_{i \neq i_{0}} O\left(\epsilon^{-2 s} \tilde{u}_{i}^{2}\right) .
\end{aligned}
$$

Hence, since $\left(W^{\prime \prime}\left(\tilde{u}_{i_{0}}\right)-W^{\prime \prime}(0)\right)=O\left(\tilde{u}_{i_{0}}\right)$, we have

$$
\begin{aligned}
I_{\epsilon}= & O\left(\tilde{u}_{i_{0}}\right)\left(\epsilon^{-2 s} \sum_{i \neq i_{0}} \tilde{u}_{i}+\tilde{\sigma}+\bar{c}_{i_{0}} \eta\right) \\
& +\sum_{i \neq i_{0}} \bar{c}_{i}\left(W^{\prime \prime}\left(\tilde{u}_{i}\right)-W^{\prime \prime}\left(\tilde{u}_{i_{0}}\right)\right) \psi_{i}+W^{\prime \prime}(0) \tilde{\sigma} \\
& +\sum_{i \neq i_{0}} \bar{c}_{i} \eta\left(W^{\prime \prime}\left(\tilde{u}_{i}\right)-W^{\prime \prime}(0)\right) \\
& -\sigma+O\left(\epsilon^{2 s}\right)+\sum_{i \neq i_{0}} O\left(\epsilon^{-2 s} \tilde{u}_{i}^{2}\right) .
\end{aligned}
$$

Now, clearly,

$$
\bar{c}_{i} \eta\left(W^{\prime \prime}\left(\tilde{u}_{i}\right)-W^{\prime \prime}(0)\right)=O\left(\tilde{u}_{i}\right) \text { and } \bar{c}_{i}\left(W^{\prime \prime}\left(\tilde{u}_{i}\right)-W^{\prime \prime}\left(\tilde{u}_{i_{0}}\right)\right) \psi_{i}=O\left(\psi_{i}\right),
$$

thus we conclude that

$$
\begin{aligned}
I_{\epsilon}= & O\left(\tilde{u}_{i_{0}}\right)\left(\epsilon^{-2 s} \sum_{i \neq i_{0}} \tilde{u}_{i}+\tilde{\sigma}+\bar{c}_{i_{0}} \eta\right) \\
& +\sum_{i \neq i_{0}} O\left(\psi_{i}\right)+W^{\prime \prime}(0) \tilde{\sigma} \\
& +\sum_{i \neq i_{0}} O\left(\tilde{u}_{i}\right)-\sigma+O\left(\epsilon^{2 s}\right)+\sum_{i \neq i_{0}} O\left(\epsilon^{-2 s} \tilde{u}_{i}^{2}\right) .
\end{aligned}
$$

This ends the proof of the desired result, since $\beta=W^{\prime \prime}(0)$ (recall (1.11)).

Now we can state the following:

Proposition 8.4. Assume that (1.2), (1.3) and (1.10) hold. Then, there exists $\delta_{0}>0$ such that, for any $0<\delta \leqslant \delta_{0}$ and $T>0$, we have

$$
\left(\bar{v}_{\epsilon}\right)_{t} \geqslant \frac{1}{\epsilon}\left(L_{s} \bar{v}_{\epsilon}-\frac{1}{\epsilon^{2 s}} W^{\prime}\left(\bar{v}_{\epsilon}\right)+\sigma\right) \quad \text { in }(0, T) \times \mathbb{R},
$$

for $\epsilon>0$ sufficiently small.

Proof. Our goal is to show that for every $x \in \mathbb{R}$

$$
I_{\epsilon} \geqslant 0
$$

for $\epsilon$ small enough: this indeed plainly implies the desired result (recall the definition of $I_{\epsilon}$ in (8.27)). 
For this, we make a preliminary observation: recalling the definition of $\tilde{u}_{i}$ in (8.26) and using Proposition 7.2, we obtain that, for any $i \in\{1, \ldots, N\}$,

$$
\left|\tilde{u}_{i}+\frac{\epsilon^{2 s}}{2 s W^{\prime \prime}(0)} \frac{x-\bar{x}_{i}(t)}{\left|x-\bar{x}_{i}(t)\right|^{1+2 s}}\right| \leqslant \frac{C \epsilon^{1+2 s}}{\left|x-\bar{x}_{i}(t)\right|^{1+2 s}} .
$$

Now we divide the proof of (8.33) by dealing with two separate cases.

Case 1: Suppose that there exists $i_{0} \in\{1, \ldots, N\}$ such that

$$
\left|x-\bar{x}_{i_{0}}(t)\right| \leqslant \epsilon^{\frac{1}{2+2 s}} .
$$

Therefore, since the $\bar{x}_{i}$ 's are well-separated, for $\epsilon$ sufficiently small we have that

$$
\left|x-\bar{x}_{i}(t)\right| \geqslant \vartheta>0, \text { for any } i \neq i_{0},
$$

where $\vartheta$ is a constant independent of $\epsilon$.

Hence, thanks to (8.34) and (8.36),

$$
\left|\sum_{i \neq i_{0}}\left(\frac{\tilde{u}_{i}}{\epsilon^{2 s}}+\frac{1}{2 s W^{\prime \prime}(0)} \frac{x-\bar{x}_{i}(t)}{\left|x-\bar{x}_{i}(t)\right|^{1+2 s}}\right)\right| \leqslant \frac{C \epsilon^{1+2 s}}{\epsilon^{2 s}} \sum_{i \neq i_{0}} \frac{1}{\left|x-\bar{x}_{i}(t)\right|^{1+2 s}} \leqslant C \epsilon .
$$

Therefore, from Lemma 8.3 we deduce that

$$
\begin{aligned}
I_{\epsilon} & =e_{\epsilon}^{i_{0}}+\beta \tilde{\sigma}-\sigma+O\left(\tilde{u}_{i_{0}}\right)\left(\eta \bar{c}_{i_{0}}+\tilde{\sigma}+\sum_{i \neq i_{0}} \frac{\tilde{u}_{i}}{\epsilon^{2 s}}\right) \\
& =e_{\epsilon}^{i_{0}}+\beta \tilde{\sigma}-\sigma+O\left(\tilde{u}_{i_{0}}\right)\left(\eta \bar{c}_{i_{0}}+\tilde{\sigma}-\frac{1}{2 s W^{\prime \prime}(0)} \sum_{i \neq i_{0}} \frac{x-\bar{x}_{i}(t)}{\left|x-\bar{x}_{i}(t)\right|^{1+2 s}}\right)+O(\epsilon) .
\end{aligned}
$$

Now, we Taylor expand the function $\frac{x-\bar{x}_{i}(t)}{\left|x-\bar{x}_{i}(t)\right|^{1+2 s}}$ for $x$ in the vicinity of the point $\bar{x}_{i_{0}}(t)$, and we use (8.35) to get

$$
\begin{aligned}
& \left|\sum_{i \neq i_{0}} \frac{x-\bar{x}_{i}(t)}{\left|x-\bar{x}_{i}(t)\right|^{1+2 s}}-\sum_{i \neq i_{0}} \frac{\bar{x}_{i_{0}}(t)-\bar{x}_{i}(t)}{\left|\bar{x}_{i_{0}}(t)-\bar{x}_{i}(t)\right|^{1+2 s}}\right| \\
& \quad=\left|\sum_{i \neq i_{0}}\left(\frac{1}{\left|\xi-\bar{x}_{i}(t)\right|^{1+2 s}}-(1+2 s) \frac{\left(\xi-\bar{x}_{i}(t)\right)^{2}}{\left|\xi-\bar{x}_{i}(t)\right|^{3+2 s}}\right)\left(x-\bar{x}_{i_{0}}(t)\right)\right| \\
& \leqslant \sum_{i \neq i_{0}} \frac{2+2 s}{\left|\xi-\bar{x}_{i}(t)\right|^{1+2 s}} \epsilon^{\frac{1}{2+2 s}} \\
& \leqslant C \epsilon^{\frac{1}{2+2 s}}
\end{aligned}
$$

where $\xi$ is a suitable point lying on the segment joining $x$ to $\bar{x}_{i_{0}}(t)$ (and hence $\left|\xi-\bar{x}_{i}(t)\right| \geqslant \vartheta / 2$ thanks to (8.35)). Therefore, using (8.38) in (8.37), we have

$$
\begin{aligned}
I_{\epsilon}= & e_{\epsilon}^{i_{0}}+\beta \tilde{\sigma}-\sigma+O\left(\tilde{u}_{i_{0}}\right)\left(\eta \bar{c}_{i_{0}}+\tilde{\sigma}-\frac{1}{2 s W^{\prime \prime}(0)} \sum_{i \neq i_{0}} \frac{\bar{x}_{i_{0}}(t)-\bar{x}_{i}(t)}{\left|\bar{x}_{i_{0}}(t)-\bar{x}_{i}(t)\right|^{1+2 s}}\right) \\
& +O(\epsilon)+O\left(\epsilon^{\frac{1}{2+2 s}}\right) .
\end{aligned}
$$


Now, we compute the term between parenthesis. From the definitions of $\eta, \bar{c}_{i_{0}}$ and $\tilde{\sigma}$ given in (5.3), (8.3) and (8.4) respectively, and recalling (1.9), we obtain

$$
\begin{aligned}
& \eta \bar{c}_{i_{0}}+\tilde{\sigma}-\frac{1}{2 s W^{\prime \prime}(0)} \sum_{i \neq i_{0}} \frac{\bar{x}_{i_{0}}(t)-\bar{x}_{i}(t)}{\left|\bar{x}_{i_{0}}(t)-\bar{x}_{i}(t)\right|^{1+2 s}} \\
= & \frac{1}{\gamma W^{\prime \prime}(0)} \dot{\bar{x}}_{i_{0}}(t)+\frac{\delta}{W^{\prime \prime}(0)}+\frac{\sigma(t, x)}{W^{\prime \prime}(0)}-\frac{1}{2 s W^{\prime \prime}(0)} \sum_{i \neq i_{0}} \frac{\bar{x}_{i_{0}}(t)-\bar{x}_{i}(t)}{\left|\bar{x}_{i_{0}}(t)-\bar{x}_{i}(t)\right|^{1+2 s}} \\
= & \frac{1}{W^{\prime \prime}(0)}\left(\frac{\overline{\bar{x}}_{i_{0}}(t)}{\gamma}+\delta+\sigma\left(t, \bar{x}_{i_{0}}(t)\right)-\frac{1}{2 s} \sum_{i \neq i_{0}} \frac{\bar{x}_{i_{0}}(t)-\bar{x}_{i}(t)}{\left|\bar{x}_{i_{0}}(t)-\bar{x}_{i}(t)\right|^{1+2 s}}\right) \\
& +\frac{\sigma(t, x)-\sigma\left(t, \bar{x}_{i_{0}}(t)\right)}{W^{\prime \prime}(0)} .
\end{aligned}
$$

Recalling (8.2), we have that

$$
\frac{\dot{\bar{x}}_{i_{0}}(t)}{\gamma}+\delta+\sigma\left(t, \bar{x}_{i_{0}}(t)\right)-\frac{1}{2 s} \sum_{i \neq i_{0}} \frac{\bar{x}_{i_{0}}(t)-\bar{x}_{i}(t)}{\left|\bar{x}_{i_{0}}(t)-\bar{x}_{i}(t)\right|^{1+2 s}}=0,
$$

and so the term in parenthesis in (8.40) vanishes. Therefore (8.40) becomes

$$
\begin{aligned}
\eta \bar{c}_{i_{0}}+\tilde{\sigma}-\frac{1}{2 s W^{\prime \prime}(0)} \sum_{i \neq i_{0}} \frac{\bar{x}_{i_{0}}(t)-\bar{x}_{i}(t)}{\left|\bar{x}_{i_{0}}(t)-\bar{x}_{i}(t)\right|^{1+2 s}} & =\frac{\sigma(t, x)-\sigma\left(t, \bar{x}_{i_{0}}(t)\right)}{W^{\prime \prime}(0)} \\
& =O\left(x-\bar{x}_{i_{0}}(t)\right) \\
& =O\left(\epsilon^{\frac{1}{2+2 s}}\right),
\end{aligned}
$$

thanks to (1.3) and (8.35). Hence (8.39) reads

$$
I_{\epsilon}=e_{\epsilon}^{i_{0}}+\beta \tilde{\sigma}-\sigma+O\left(\epsilon^{\frac{1}{2+2 s}}\right)+O(\epsilon)+O\left(\epsilon^{\frac{1}{2+2 s}}\right) .
$$

Also, in the light of (8.4), we see that

$$
\beta \tilde{\sigma}-\sigma=\delta>0 .
$$

Now, we claim that

(8.43) the error $e_{\epsilon}^{i_{0}}$ (that was defined in (8.28)) tends to zero as $\epsilon \rightarrow 0$.

For this, we notice that $\psi_{i}=\psi\left(\frac{x-\bar{x}_{i}(t)}{\epsilon}\right)$, with $i \neq i_{0}$, tends to zero because of the behavior of the corrector at infinity (recall (5.2) and (8.36)). Moreover, thanks to (5.1) and (8.36) we have that, for $i \neq i_{0}$,

$$
\tilde{u}_{i}=u\left(\frac{x-\bar{x}_{i}(t)}{\epsilon}\right)-H\left(\frac{x-\bar{x}_{i}(t)}{\epsilon}\right)=O\left(\frac{\epsilon^{2 s}}{\left|x-\bar{x}_{i}(t)\right|^{2 s}}\right)=O\left(\epsilon^{2 s}\right)
$$

and

$$
\frac{\left(\tilde{u}_{i}\right)^{2}}{\epsilon^{2 s}}=\frac{O\left(\epsilon^{4 s}\right)}{\epsilon^{2 s}}=O\left(\epsilon^{2 s}\right),
$$

thus proving (8.43).

Hence, from (8.41), (8.42) and (8.43) we obtain that for $\epsilon$ sufficiently small

$$
I_{\epsilon} \geqslant \frac{\delta}{2}>0
$$

which implies (8.33) in this case. 
Case 2: Suppose that $\left|x-\bar{x}_{i}(t)\right|>\epsilon^{\frac{1}{2+2 s}}$ for every $i \in\{1, \ldots, N\}$. In this case, we can fix $i_{0}$ arbitrarily, say $i_{0}:=1$ for concreteness. We use (8.34) to obtain

$$
\begin{aligned}
\left|\sum_{i \neq i_{0}}\left(\frac{\tilde{u}_{i}}{\epsilon^{2 s}}+\frac{1}{2 s W^{\prime \prime}(0)} \frac{x-\bar{x}_{i}(t)}{\left|x-\bar{x}_{i}(t)\right|^{1+2 s}}\right)\right| & \leqslant \frac{C \epsilon^{1+2 s}}{\epsilon^{2 s}} \sum_{i \neq i_{0}} \frac{1}{\left|x-\bar{x}_{i}(t)\right|^{1+2 s}} \\
& \leqslant C \frac{\epsilon}{\epsilon^{\frac{1+2 s}{2+2 s}}}=C \epsilon^{\frac{1}{2+2 s}} .
\end{aligned}
$$

Therefore, from Lemma 8.3 and the definition of $\tilde{\sigma}$ in (8.4) we have

$$
I_{\epsilon}=e_{\epsilon}^{i_{0}}+\delta+O\left(\tilde{u}_{i_{0}}\right)\left(\eta \bar{c}_{i_{0}}+\tilde{\sigma}-\frac{1}{2 s W^{\prime \prime}(0)} \sum_{i \neq i_{0}} \frac{x-\bar{x}_{i}(t)}{\left|x-\bar{x}_{i}(t)\right|^{1+2 s}}\right)+O\left(\epsilon^{\frac{1}{2+2 s}}\right) .
$$

We notice that, for any $i \neq i_{0}$,

$$
\left|\frac{x-\bar{x}_{i}(t)}{\left|x-\bar{x}_{i}(t)\right|^{1+2 s}}\right| \leqslant \frac{1}{\left|x-\bar{x}_{i}(t)\right|^{2 s}} \leqslant \frac{1}{\epsilon^{\frac{2 s}{2+2 s}}}=O\left(\epsilon^{-\frac{s}{1+s}}\right) .
$$

Notice that this term is divergent as $\epsilon$ tends to zero. From (8.45) we conclude that

$$
\eta \bar{c}_{i_{0}}+\tilde{\sigma}-\frac{1}{2 s W^{\prime \prime}(0)} \sum_{i \neq i_{0}} \frac{x-\bar{x}_{i}(t)}{\left|x-\bar{x}_{i}(t)\right|^{1+2 s}}=O\left(\epsilon^{-\frac{s}{1+s}}\right)
$$

since the other terms are bounded. By plugging this into (8.44) we obtain

$$
I_{\epsilon}=e_{\epsilon}^{i_{0}}+\delta+O\left(\tilde{u}_{i_{0}}\right) \cdot O\left(\epsilon^{-\frac{s}{1+s}}\right)+O\left(\epsilon^{\frac{1}{2+2 s}}\right) .
$$

Now we observe that for every $i \in\{1, \ldots, N\}$,

$$
\tilde{u}_{i}=u\left(\frac{x-\bar{x}_{i}(t)}{\epsilon}\right)-H\left(\frac{x-\bar{x}_{i}(t)}{\epsilon}\right)=O\left(\frac{\epsilon^{2 s}}{\left|x-\bar{x}_{i}(t)\right|^{2 s}}\right)=O\left(\frac{\epsilon^{2 s}}{\epsilon^{\frac{2 s}{2+2 s}}}\right)=O\left(\epsilon^{\frac{s(1+2 s)}{1+s}}\right) .
$$

As a consequence

$$
\frac{\left(\tilde{u}_{i}\right)^{2}}{\epsilon^{2 s}}=O\left(\epsilon^{\frac{2 s^{2}}{1+s}}\right) \text { and } O\left(\tilde{u}_{i_{0}}\right) \cdot O\left(\epsilon^{-\frac{s}{1+s}}\right)=O\left(\epsilon^{\frac{2 s^{2}}{1+s}}\right) .
$$

By inserting this into (8.46) we get

$$
I_{\epsilon}=e_{\epsilon}^{i_{0}}+\delta+O\left(\epsilon^{\alpha}\right)
$$

for some $\alpha>0$. Now we check that

$$
\text { the error term } e_{\epsilon}^{i_{0}} \text { tends to zero as } \epsilon \rightarrow 0 \text {. }
$$

For this, we remark that, in this case,

$$
\frac{\left|x-\bar{x}_{i}(t)\right|}{\epsilon} \geqslant \frac{\epsilon^{\frac{1}{2+2 s}}}{\epsilon}=\epsilon^{-\frac{1+2 s}{2+2 s}},
$$

which diverges for small $\epsilon$. Therefore, for $x$ fixed as in the assumption of Case 2, we have that

$$
\psi_{i}(x)=\psi\left(\frac{x-\bar{x}_{i}(t)}{\epsilon}\right) \longrightarrow 0
$$

as $\epsilon \rightarrow 0$, due to the infinitesimal behavior of $\psi$ at infinity (see (5.2)). Using this, (8.47), (8.48) and the definition of the error term given in (8.28), we obtain (8.50). 
Hence, by using (8.50) inside (8.49) and recalling that $\delta>0$, we conclude that

$$
I_{\epsilon} \geqslant \frac{\delta}{2}>0 \text {, }
$$

for $\epsilon$ sufficiently smooth, thus proving (8.33) in this case too.

8.2. Existence and uniqueness of the solution. We observe that, for $\epsilon$ sufficiently small, the initial condition $v_{\epsilon}^{0}$ given in (1.10) satisfies

$$
-1 \leqslant v_{\epsilon}^{0} \leqslant N+1 \text {. }
$$

Moreover, setting

$$
K_{\epsilon}:=\frac{1}{\epsilon^{1+2 s}}\left\|W^{\prime}\right\|_{L^{\infty}(\mathbb{R})}+\frac{1}{\epsilon}\|\sigma\|_{L^{\infty}(\mathbb{R})},
$$

we have that the functions

$$
\underline{u}_{\epsilon}(t, x):=-1-K_{\epsilon} t \quad \text { and } \bar{u}_{\epsilon}(t, x):=N+1+K_{\epsilon} t
$$

are respectively sub and supersolutions of $(1.4)$ in $[0,+\infty) \times \mathbb{R}$. Hence, there exists a unique, continuous, solution $v_{\epsilon}$ of $(1.4)$ in $[0,+\infty) \times \mathbb{R}$, thanks to the Perron's method and the comparison principle (see [4]).

8.3. Convergence. In Subsection 8.1 we constructed a supersolution $\bar{v}_{\epsilon}$ of (1.4) in $[0, T) \times \mathbb{R}$. In a similar way, one can build also a subsolution $\underline{v}_{\epsilon}$ (defined as $\bar{v}_{\epsilon}$ in $(8.5)$ but with $\delta<0$ ). Notice that, from Proposition 8.2 (and its analogue for $\underline{v}_{\epsilon}$ ), we have that at the initial time the following holds for every $x \in \mathbb{R}$ :

$$
\underline{v}_{\epsilon}(0, x) \leqslant v_{\epsilon}(0, x) \leqslant \bar{v}_{\epsilon}(0, x) .
$$

Then, from the comparison principle, we obtain

$$
\underline{v}_{\epsilon}(t, x) \leqslant v_{\epsilon}(t, x) \leqslant \bar{v}_{\epsilon}(t, x), \quad t \in[0, T), \quad x \in \mathbb{R} .
$$

Passing to the limit as $\epsilon \rightarrow 0$, using the continuity of $v_{\epsilon}$ and recalling Lemma 8.1 (and its analogue for $\underline{v}_{\epsilon}$ ), and taking $\delta$ as small as we wish in the end, we get (1.13) and (1.14) in $[0, T) \times \mathbb{R}$ for any fixed $T>0$, and thus in $[0,+\infty) \times \mathbb{R}$ since $T$ is arbitrary. This concludes the proof of Theorem 1.1.

\section{REFERENCES}

[1] R. Alicandro, L. De Luca, A. Garroni, M. Ponsiglione: Metastability and dynamics of discrete topological singularities in two dimensions: a $\Gamma$-convergence approach. Preprint. Available at http://cvgmt.sns.it/paper/2215/

[2] O. Alvarez, P. Hoch, Y. Le Bouar, R. Monneau: Dislocation dynamics: short-time existence and uniqueness of the solution. Arch. Ration. Mech. Anal. 181 (2006), no. 3, 449-504.

[3] B. Barrios, A. Figalli, E. VAldinoci: Bootstrap regularity for integro-differential operators and its application to nonlocal minimal surfaces. Ann. Scu. Norm. Sup. Pisa, to appear. DOI : 0.2422/2036-2145.201202_007.

[4] G. Barles, C. Imbert: Second-order elliptic integro-differential equations: viscosity solutions' theory revisited. Ann. Inst. H. Poincaré Anal. Non Linéaire 25 (2008), no. 3, 567-585.

[5] X. Cabré, Y. Sire: Nonlinear equations for fractional Laplacians II: existence, uniqueness, and qualitative properties of solutions. Trans. Amer. Math. Soc., to appear. Available at http://arxiv.org/abs/1111.0796 
[6] F. Da Lio, N. Forcadel, R. Monneau: Convergence of a non-local eikonal equation to anisotropic mean curvature motion. Application to dislocation dynamics. J. Eur. Math. Soc. (JEMS) 10 (2008), no. 4, 1061-1104.

[7] C. Denoual: Dynamic dislocation modeling by combining Peierls Nabarro and Galerkin methods. Phys. Rev. B $\mathbf{7 0}$ (2004), 024106.

[8] E. Di Nezza, G. Palatucci, E. Valdinoci: Hitchhiker's guide to the fractional Sobolev spaces. Bull. Sci. math. 136 (2012), no. 5, 521-573.

[9] N. Forcadel, C. Imbert, R. Monneau: Homogenization of some particle systems with twobody interactions and of the dislocation dynamics. Discrete Contin. Dyn. Syst. 23 (2009), no. $3,785-826$.

[10] A. Garroni, S. Müller: Г-limit of a phase-field model of dislocations. SIAM J. Math. Anal. 36 (2005), no. 6, 1943-1964.

[11] M. D. M. González, R. Monneau: Slow motion of particle systems as a limit of a reactiondiffusion equation with half-Laplacian in dimension one. Discrete Contin. Dyn. Syst. 32 (2012), no. $4,1255-1286$.

[12] C. P. Grant: Slow motion in one-dimensional Cahn-Morral systems. SIAM J. Math. Anal. 26 (1995), no. 1, 21-34.

[13] J. P. Hirth, J. Lothe: Theory of Dislocations. Krieger Publishing Company (1991), pp. 872.

[14] C. ImBert: Level set approach for fractional mean curvature flows. Interfaces Free Bound. 11 (2009), 153-176.

[15] G. Lu: The Peierls-Nabarro model of dislocations: a venerable theory and its current development. In S. YIP, Ed.: Handbook of Materials Modeling, Springer Netherlands (2005), 793-811.

[16] R. Monneau, S. Patrizi: Homogenization of the Peierls-Nabarro model for dislocation dynamics. J. Differential Equations 253 (2012), no. 7, 2064-2105.

[17] A. B. Movchan, R. Bullough, J. R. Willis: Stability of a dislocation: discrete model. European J. Appl. Math. 4 (1998), no. 8, 373-396.

[18] F. R. N. Nabarro: Fifty-year study of the Peierls-Nabarro stress. Mat. Sci. Eng. A 234236 (1997), 67-76.

[19] G. Palatucci, O. Savin, E. Valdinoci: Local and global minimizers for a variational energy involving a fractional norm. Ann. Mat. Pura Appl. (4) 192 (2013), no. 4, 673-718.

[20] R. Servadei, E. Valdinoci: Weak and viscosity solutions of the fractional Laplace equation. Publ. Mat. 58 (2014), no. 1.

[21] L. Silvestre: Regularity of the obstacle problem for a fractional power of the Laplace operator. Ph.D. Thesis, University of Texas at Austin (2005).

[22] L. Silvestre: Regularity of the obstacle problem for a fractional power of the Laplace operator. Comm. Pure Appl. Math. 60 (2007), no. 1, 67-112.

[23] J. F. Toland: The Peierls-Nabarro and Benjamin-Ono equations. J. Funct. Anal. 145 (1997), no. 1, 136-150.

[24] H. Wei, Y. Xiang, P. Ming: A generalized Peierls-Nabarro model for curved dislocations using discrete Fourier transform. Commun. Comput. Phys. 4 (2008), no. 2, 275-293. 
(Serena Dipierro) School of Mathematics, University of Edinburgh, King's Buildings, Mayfield Road, Edinburgh EH9 3JZ, Scotland, UK

E-mail address: serydipierro@yahoo.it

(Giampiero Palatucci) SISSA - International School for Advanced Studies, Via Bonomea 256, 34136 Trieste, Italy

E-mail address: giampiero.palatucci@sissa.it

(Enrico Valdinoci) Weierstrass Institut für Angewandte Analysis und Stochastik, Hausvogteiplatz 11A, 10117 Berlin, Germany

E-mail address: enrico.valdinoci@wias-berlin.de 